\title{
Pharmacokinetics and disposition of monoterpene glycosides derived from Paeonia lactiflora roots (Chishao) after intravenous dosing of antiseptic XueBiJing injection in human subjects and rats
}

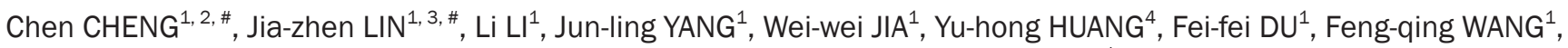
Mei-juan $\mathrm{LI}^{1}$, Yan-fen $\mathrm{LI}^{4}$, Fang XU ${ }^{1}$, Na-ting ZHANG ${ }^{1}$, Olajide E. OLALEYE ${ }^{1}$, Yan SUN ${ }^{1,}{ }^{\dagger}$, Jian $\mathrm{LI}^{1}$, Chang-hai SUN ${ }^{5}$, Gui-ping ZHANG $^{5}$, Chuan $\mathrm{LI}^{1,2,3, *}$

\begin{abstract}
${ }^{1}$ Shanghai Institute of Materia Medica, Chinese Academy of Sciences, Shanghai 201203, China; ${ }^{2}$ Institute of Chinese Materia Medica, China Academy of Chinese Medical Sciences, Beijing 100700, China; ${ }^{3}$ University of Chinese Academy of Sciences, Shanghai 201203, China; ${ }^{4}$ Second Affiliated Hospital, Tianjin University of Traditional Chinese Medicine, Tianjin 300150, China; ${ }^{5}$ Tianjin Chasesun Pharmaceutical Co, Ltd, Tianjin 301700, China
\end{abstract}

\begin{abstract}
Aim: Monoterpene glycosides derived from Paeonia lactiflora roots (Chishao) are believed to be pharmacologically important for the antiseptic herbal injection XueBiJing. This study was designed to characterize the pharmacokinetics and disposition of monoterpene glycosides.

Methods: Systemic exposure to Chishao monoterpene glycosides was assessed in human subjects receiving an intravenous infusion and multiple infusions of XueBiJing injection, followed by assessment of the pharmacokinetics of the major circulating compounds. Supportive rat studies were also performed. Membrane permeability and plasma-protein binding were assessed in vitro. Results: A total of 18 monoterpene glycosides were detected in XueBiJing injection (content levels, $0.001-2.47 \mathrm{mmol} / \mathrm{L}$ ), and paeoniflorin accounted for $85.5 \%$ of the total dose of monoterpene glycosides detected. In human subjects, unchanged paeoniflorin exhibited considerable levels of systemic exposure with elimination half-lives of 1.2-1.3 h; no significant metabolite was detected. Oxypaeoniflorin and albiflorin exhibited low exposure levels, and the remaining minor monoterpene glycosides were negligible or undetected. Glomerular-filtration-based renal excretion was the major elimination pathway of paeoniflorin, which was poorly bound to plasma protein. In rats, the systemic exposure level of paeoniflorin increased proportionally as the dose was increased. Rat lung, heart, and liver exposure levels of paeoniflorin were lower than the plasma level, with the exception of the kidney level, which was 4.3-fold greater than the plasma level; brain penetration was limited by the poor membrane permeability.

Conclusion: Due to its significant systemic exposure and appropriate pharmacokinetic profile, as well as previously reported antiseptic properties, paeoniflorin is a promising XueBiJing constituent of therapeutic importance.
\end{abstract}

Keywords: herbal injection; XueBiJing; Paeonia lactiflora roots; Chishao; monoterpene glycosides; paeoniflorin; pharmacokinetics; disposition

Acta Pharmacologica Sinica (2016) 37: 530-544; doi: 10.1038/aps.2015.103; published online 1 Feb 2016

\section{Introduction}

Sepsis is a common and potentially fatal systemic illness trig-

\footnotetext{
\# These authors contributed equally to this work.

${ }^{\dagger}$ Now in Laboratory of Phase I Clinical Trials, Fudan University Shanghai Cancer Center, Shanghai 200032, China

* To whom correspondence should be addressed.

E-mail chli@simm.ac.cn

Received 2015-04-27 Accepted 2015-09-18
}

gered by microbial infection, and it often leads to the impaired function of vital organs, including the lungs, heart, brain, kidneys, and/or liver ${ }^{[1]}$. Conventional management of sepsis includes an initial management bundle that is administered within six hours after the patient's presentation and a second management bundle that is administered in the intensive care unit ${ }^{[2]}$. The initial management bundle provides cardiorespiratory resuscitation and mitigates the immediate threat of uncontrolled infection. Resuscitation requires the use of 
intravenous fluids and vasopressors, with oxygen therapy and mechanical ventilation provided as necessary. The initial management of infection requires initiating broad-spectrum and timely antibiotic therapy ${ }^{[3]}$. After the first six hours, attention is focused on monitoring and supporting organ function, avoiding complications, and de-escalating the initial antibiotic therapy when possible. For patients with hypotension or hyperlactatemia, immunomodulatory therapy is advocated, which is often given as a short course of hydrocortisone. Although many therapeutic agents for the treatment of sepsis have been evaluated, no specific antiseptic agent has been approved $^{[4]}$.

In China, XueBijing injection is extensively used as an addon therapy in the conventional management of sepsis. This herbal injection, available as a sterile, nonpyrogenic parenteral dosage form for intravenous administration, was approved in 2004 by the China Food and Drug Administration (China FDA; Beijing, China) for the treatment of sepsis and multiple organ dysfunction syndromes. Each milliliter of the injection is prepared from a combination of $0.1 \mathrm{~g}$ each of Carthamus tinctorius flowers (Honghua in Chinese), Paeonia lactiflora roots (Chishao), Ligusticum chuanxiong rhizomes (Chuanxiong), Angelica sinensis roots (Danggui), and Salvia miltiorrhiza roots (Danshen), yielding an herb-to-injection ratio of 1:2. Several meta-analyses of clinical trials show that XueBijing therapy is associated with a reduced $28-\mathrm{d}$ mortality rate, improved acute physiology and chronic health evaluation II score, shortened stay in the intensive care unit, decreased ventilation time, reduced incidence of complications, and improved patient outcome; it appears to have a low incidence of side effects $^{[5-8]}$. The herbal injection improves microcirculation, protects the endothelium, alleviates inflammation, and regulates the immune response. Research on XueBiJing therapy may advance the understanding of life-threatening sepsis and result in further lowered mortality rates and improved patient prognoses. To this end, it is important to identify the active principles responsible for the therapeutic actions of the herbal injection.

Herbal medicines normally contain multiple chemical constituents. It is hypothesized that the active principles responsible for the therapeutic effects of an herbal medicine come from a few constituents with favorable drug-like properties, including the desired pharmacologic potency, a wide safety margin, appropriate pharmacokinetic (PK) properties, and adequate content in the dosed medicine ${ }^{[9,10]}$. Recent multicompound PK studies of herbal medicines indicate notable differences between the circulating herbal compounds and the chemical ingredients of the dosed medicine ${ }^{[9,11-17]}$. Pharmacologically active herbal compounds that exhibit considerable levels of body exposure after dosing an herbal medicine are the most likely to form the basis of therapeutic efficacy of the medicine. Accordingly, PK research on XueBijing injection is a key step with which to identify the active principles responsible for its antiseptic properties.

As a part of our ongoing PK research on XueBijing injection, the current study focused on monoterpene glycosides originat- ing from Chishao. Several Chishao monoterpene glycosides, including paeoniflorin, albiflorin, oxypaeoniflorin, benzoylpaeoniflorin, and galloylpaeoniflorin, have been detected in XueBijing injection ${ }^{[18]}$. Cell- and animal-based studies have shown that paeoniflorin and other monoterpene glycosides exhibit anti-inflammatory, antiplatelet, antithrombosis, antioxidative, and neuroprotective properties ${ }^{[19-33]}$. Due to these pharmacological properties, several bioanalytical assays were developed to measure plasma concentrations of paeoniflorin and albiflorin ${ }^{[34-36]}$. However, PK data regarding the monoterpene glycosides remain quite limited, particularly human PK data. Paeoniflorin and albiflorin were reported to have low oral bioavailability in rats $(\leq 5 \%)^{[37,38]}$. Poor membrane permeability, P-glycoprotein-mediated efflux, and intestinalmicroflora-induced metabolism were considered to contribute to the poor oral bioavailability of paeoniflorin in rats ${ }^{[39,40]}$. The metabolism of oral paeoniflorin, which was induced by human and rat intestinal microflora, resulted in the formation of paeonimetabolin I, paeonimetabolin II, and paeoniflorgenin ${ }^{[41-44]}$. Recently, more metabolites of paeoniflorin were detected in rat plasma and urine after oral dosing of paeoniflorin or a Chishao extract ${ }^{[45]}$, but their chemistry was not fully characterized. The current study was designed to investigate the pharmacokinetics and disposition of major circulating Chishao monoterpene glycosides after intravenous dosing of XueBijing injection in human subjects and rats. The information gained here will facilitate the identification of the active principles responsible for the therapeutic actions of the injection and thus help to achieve optimal herbal therapy.

\section{Materials and methods}

\section{XueBiJing injection and Paeonia lactiflora roots}

XueBijing injection used in the current study was manufactured by Tianjin Chasesun Pharmaceutical Co, Ltd (Tianjin, China) with a China FDA ratification number of GuoYaoZhunZi-Z20040033 for market approval as a drug product. Supplementary Information Appendix S1 briefly describes the method for the preparation of XueBijing injection, which is standardized to contain a $1.0-1.7 \mathrm{mg} / \mathrm{mL}$ concentration of paeoniflorin and a $0.2-0.5 \mathrm{mg} / \mathrm{mL}$ concentration of hydroxysafflor yellow A. Samples of eight lots (1309271, 1309281, 1309291, 1309301, 1405301, 1406161, 1408191, and 1410081) of XueBiJing injection were obtained from Tianjin Chasesun Pharmaceutical for analysis of their chemical composition and quality consistency with respect to Chishao monoterpene glycosides. XueBijing injection from the lot 1309301 was used in the current human and rat studies. Samples of three lots $(1205224,1404111$, and 1501041) of P lactiflora roots (Chishao) were also obtained from Tianjin Chasesun Pharmaceutical.

\section{Chemicals and reagents}

Reference standards of albiflorin, benzoylpaeoniflorin, mudanpioside $\mathrm{C}$, oxypaeoniflorin, and paeoniflorin were purchased from Tauto Biotech (Shanghai, China). Benzoyloxypaeoniflorin, desbenzoylpaeoniflorin, galloylpaeoniflorin, and mudan- 
pioside J were purchased from BioBioPha (Kunming, China). The purity of these compounds was $\geq 98 \%$. HPLC-grade organic solvents were obtained from Sinopharm Chemical Reagent Co, Ltd (Shanghai, China). HPLC-grade water was prepared in-house with a Millipore Milli-Q Integral 3 cabinet water purifying system (Bedford, MA, USA).

Sodium heparin and isoflurane were also obtained from Sinopharm Chemical Reagent Co, Ltd. Pentobarbital was obtained from Shanghai Westang Biotechnology (Shanghai, China). Dulbecco's modified Eagle's medium, penicillinstreptomycin, and minimal essential medium nonessential amino acids were obtained from Gibco Invitrogen Cell Culture (Grand Island, NY, USA). Fetal bovine serum was purchased from HyClone Laboratories (Logan, UT, USA). Hanks' balanced salt solution, taurocholic acid, antipyrine, atenolol, rhodamine 123, sulfasalazine, verapamil, indomethacin, and novobiocin were obtained from Sigma-Aldrich (St Louis, MO, USA).

\section{Human study}

The protocol for the human PK study of XueBijing injection was reviewed and approved by the Ethics Committee of Clinical Investigation at the Second Affiliated Hospital of Tianjin University of Traditional Chinese Medicine (Tianjin, China). The human study was registered in the Chinese Clinical Trials Registry (www.chictr.org.cn) with a registration number of ChiCTR-ONRC-13003932 prior to its initiation at the National Clinical Research Center of the hospital. Healthy volunteers (18 men and 18 women) aged between 19 and 31 years old with body mass indexes of $19.0-23.9 \mathrm{~kg} / \mathrm{m}^{2}$ provided written informed consent to participate in the study. The human subjects were considered to be in good health on the basis of medical history, physical examination, vital signs measurement, electrocardiogram, and clinical laboratory tests. They were required to be nonsmokers and not to be allergic to XueBijing injection. The female volunteers were negative for menstruation and pregnancy. Synthetic drugs and herbal products were prohibited beginning two weeks prior to the commencement of the study and continuing until the end of the study period. Alcoholic beverages were also prohibited beginning two days prior to the commencement of the study and continuing until the end of the study period.

The human subjects were randomly assigned into three groups (six male and six female subjects in each group). According to the label doses for clinical use $(50 \mathrm{~mL} /$ person for treatment of sepsis and $100 \mathrm{~mL} /$ person for treatment of multiple organ dysfunction syndromes), each subject received a single intravenous infusion of XueBijing injection using a ZNB-XB intelligent infusion pump (Beijing Kelifeng, Beijing, China). The subjects were given one of the three following dosage regimens: a 75-min infusion of $100 \mathrm{~mL}$ of the herbal injection diluted in $100 \mathrm{~mL}$ of $0.9 \%$ sodium chloride $(\mathrm{NaCl})$ injection (GuoYaoZhunZi-H12020025; China Otsuka Pharmaceutical Co, Ltd, Tianjin, China) (group A), a 150-min infusion of $100 \mathrm{~mL}$ of the herbal injection diluted in $200 \mathrm{~mL}$ of the $\mathrm{NaCl}$ injection (group B), and a 75-min infusion of $50 \mathrm{~mL}$ of the herbal injection diluted in $100 \mathrm{~mL}$ of the $\mathrm{NaCl}$ injection (group C). Serial blood samples (approximately $3 \mathrm{~mL}$ ) were collected, into heparinized tubes, from an antecubital vein catheter before and 0.17, 0.5, 1.25 (75 min), 1.42 (groups A and $\mathrm{C}$ only), 1.75 (groups $\mathrm{A}$ and $\mathrm{C}$ only), 2.25 (groups $\mathrm{A}$ and C only), 2.5 (150 min; group B only), 2.67 (group B only), 3 (group B only), 3.25 (groups A and C only), 3.5 (group B only), 4.5 (group B only), 5.25 (groups A and C only), 6.5 (group B only), 9.25 (groups A and C only), 10.5 (group B only), and 24 $\mathrm{h}$ after starting the infusion. Serial urine samples were also collected before and $0-3,3-6,6-10$, and 10-24 h after starting the infusion. In addition, the six male subjects of group $C$ continued to receive the same dose of the herbal injection each day for the following six days. On d 2-6, blood sampling was performed before and $1.25 \mathrm{~h}(75 \mathrm{~min})$ after the daily infusion was started; on d 7, blood and urine samples were collected according to the time schedules for $\mathrm{d} 1$. The collected blood samples were centrifuged at $3000 \times g$ for $5 \mathrm{~min}$ to obtain plasma fractions, which were stored at $-70^{\circ} \mathrm{C}$ pending analysis. After collection, the urine samples were immediately weighed and stored at $-70^{\circ} \mathrm{C}$ without use of any preservative. Serum alanine aminotransferase, aspartate aminotransferase, total protein, albumin/globulin ratio, total bilirubin, and direct bilirubin were monitored as liver function markers for the human subjects; serum creatinine and blood urea nitrogen were also assessed to monitor human kidney function. Liver and kidney functions were monitored before and $24 \mathrm{~h}$ after starting the infusion; subjects receiving multiple doses were also monitored on $\mathrm{d} 4$ (before starting the infusion) and $\mathrm{d} 7$ (before and $24 \mathrm{~h}$ after starting the infusion).

\section{Rat studies}

A total of 44 male Sprague-Dawley rats (230-270 g) were obtained from Sino-British SIPPR/BK Laboratory Animal (Shanghai, China). The rat studies were conducted in compliance with the Guidance for Ethical Treatment of Laboratory Animals (Ministry of Science and Technology of China, 2006) and according to protocols that were reviewed and approved by the Institutional Animal Care and Use Committee at the Shanghai Institute of Materia Medica (Shanghai, China). Rats were maintained in a $20-24{ }^{\circ} \mathrm{C}$ room with unidirectional airflow, relative humidity between $30 \%$ and $70 \%$, and a $12-\mathrm{h}$ light/dark cycle. Rats were given filtered tap water and commercial rat chow ad libitum and allowed to acclimate to the facilities and environment for three days prior to the study. Rats received in-house femoral artery cannulation for blood sampling or bile duct cannulation for bile sampling ${ }^{[15]}$. The surgical rats were allowed to regain their preoperative body weight prior to the study and were euthanized with $\mathrm{CO}_{2}$ gas after the study.

In the first study, conscious and freely moving rats were randomly divided into three groups of four rats each and received a single 30-min intravenous infusion of XueBijing injection at 5, 10, and $20 \mathrm{~mL} / \mathrm{kg}$ via PHD 2000 infusion pumps (Harvard Apparatus, Holliston, MA, USA). The doses of 5 and $10 \mathrm{~mL} / \mathrm{kg}$ for the rats were derived from the label dose 
of XueBiJing injection for patients with sepsis $(50 \mathrm{~mL} /$ person) and those with multiple organ dysfunction syndromes (100 $\mathrm{mL} /$ person), respectively, according to dose normalization by body surface area ${ }^{[46]}$. The rat dose of $20 \mathrm{~mL} / \mathrm{kg}$ was four and two times as much as the label doses, respectively. Serial blood samples (approximately $80 \mu \mathrm{L}$ ) were collected, into heparinized tubes, before and $0.17,0.33,0.5$ (30 $\mathrm{min}), 0.58,0.75,1$, $1.5,2.5,4.5,6.5,8.5,10.5$, and $24 \mathrm{~h}$ after starting the infusion. The blood samples were centrifuged to produce the plasma fractions.

In the second study, four rats were housed singly in metabolic cages, and the urine collection tubes were frozen at $-15^{\circ} \mathrm{C}$ during sampling. Urine samples were collected before and $0-4,4-8$, and $8-24 \mathrm{~h}$ after a single 30-min intravenous infusion of XueBijing injection at $10 \mathrm{~mL} / \mathrm{kg}$ and were weighed.

In the third study, four rats received a single 30-min intravenous infusion of XueBijing injection at $10 \mathrm{~mL} / \mathrm{kg}$. Bile samples were collected before and 0-2, 2-4, 4-8, and 8-24 h after dosing and were weighed. A sodium taurocholate solution $(1.5 \mathrm{~mL} / \mathrm{h} ; \mathrm{pH} 7.4)$ was infused into the duodenum during bile collection.

In the fourth study, rats under isoflurane anesthesia were killed by bleeding from the abdominal aorta at $0.08,0.25,0.5$, and $1 \mathrm{~h}$ (four rats per time point) after dosing a single intravenous bolus of XueBijing injection at $10 \mathrm{~mL} / \mathrm{kg}$ from the tail veins. The blood samples were heparinized and centrifuged to produce the plasma fractions. Selected tissues, including the heart, lungs, brain, liver, and kidneys, were excised, rinsed in ice-cold saline, blotted, weighed, and homogenized in fourfold volumes of ice-cold saline.

In the fifth study, rats were randomized into two groups of four rats each. They received a single intravenous bolus dose of XueBijing injection at $10 \mathrm{~mL} / \mathrm{kg}$ or a single intravenous bolus dose of purified paeoniflorin (dissolved in the vehicle of XueBijing injection) at $11.4 \mathrm{mg} / \mathrm{kg}$ through the tail veins. Serial blood samples (approximately $80 \mu \mathrm{L}$ ) were collected, into heparinized tubes, before and $0.08,0.25,0.5,1,2,4,6,8$, 10 , and $24 \mathrm{~h}$ after dosing. The blood samples were centrifuged to produce the plasma fractions.

All rat samples were stored at $-70^{\circ} \mathrm{C}$ pending analysis.

\section{Determination of plasma protein binding}

A rapid ultrafiltration method by Guo et al ${ }^{[47]}$ was used to assess fractions of plasma-protein-unbound compound $\left(f_{\mathrm{u}}\right)$. The test compounds paeoniflorin, oxypaeoniflorin, and albiflorin were individually added into blank human and rat plasma to generate nominal concentrations of 1 and $10 \mu \mathrm{mol} / \mathrm{L}$. Microcon YM-30 centrifugal filter devices (Bedford, MA, USA) were used to load the samples and centrifuged at $13362 \times g$ and $37^{\circ} \mathrm{C}$ for $3 \mathrm{~min}$. Nonspecific binding of the test compounds to the Microcon filter membrane was negligible.

\section{Transport study in Caco-2 cell monolayers}

Caco-2 cells (American Type Culture Collection, Manassas, VA, USA) were cultured as previously described ${ }^{[48]}$. Bidirectional transport experiments were conducted in triplicate at
$10 \mu \mathrm{mol} / \mathrm{L}$ for paeoniflorin, oxypaeoniflorin, and albiflorin in Hanks' balanced salt solution. Only those Caco-2 cell monolayers that exhibited a transepithelial electrical resistance of $400-500 \Omega \cdot \mathrm{cm}^{2}$ were used. The applicability of the cell monolayers was also evaluated using antipyrine, atenolol, rhodamine 123, sulfasalazine, verapamil, indomethacin, and novobiocin prior to use. Samples were collected from the receiver compartment 60 and $120 \mathrm{~min}$ after the initiation of incubation; samples were also collected from the donor compartment at $120 \mathrm{~min}$. The apparent permeability coefficient $\left(P_{\text {app }}\right)$ expressed in $\mathrm{cm} / \mathrm{s}$ was calculated using the following equation:

$$
P_{\text {app }}=(\Delta Q / \Delta t) /\left(A \times C_{0}\right)
$$

where $\Delta Q / \Delta t$ is the linear appearance rate of the test compounds on the receiver side in $\mu \mathrm{mol} / \mathrm{s} ; A$ is the surface area of the cell monolayer in $\mathrm{cm}^{2}$; and $C_{0}$ is the initial concentration of the test compound on the donor compartment in $\mu \mathrm{mol} / \mathrm{L}$. According to in-house Caco-2 cell monolayer data, compounds with $P_{\text {app }}$ values $<0.2 \times 10^{-6}, 0.2 \times 10^{-6}-2.8 \times 10^{-6}$, and $>2.8 \times 10^{-6}$ $\mathrm{cm} / \mathrm{s}$ were defined to represent low, intermediate, and high permeability, respectively ${ }^{[12]}$. An efflux ratio $\left(P_{\text {app, B-A }} / P_{\text {app,A- }}\right.$ в) was used to implicate possible involvement of transportermediated efflux activity, and an efflux ratio $>3$ was considered to be a positive result.

\section{Analysis of Chishao monoterpene glycosides and the metabolites} A Waters Synapt G2 high-definition time-of-flight mass spectrometer (TOF-MS; Manchester, UK), interfaced via a Zspray/LockSpray ESI source with a Waters Acquity UPLC separation module (Milford, MA, USA), was used to analyze Chishao monoterpene glycosides and their metabolites. The mass spectrometer was operated in resolution mode with a resolving power of approximately 20000 , and the ESI source was operated in the negative ion mode. The mass spectrometer parameters were optimized in a similar manner to that previously described ${ }^{[16]}$. Chromatographic separations were achieved on a Waters Acquity UPLC BEH C18 1.7- $\mu \mathrm{m}$ column $\left(100 \mathrm{~mm} \times 2.1 \mathrm{~mm}\right.$ id; Dublin, Ireland; kept at $\left.40^{\circ} \mathrm{C}\right)$ using a mobile phase that consisted of solvent A (methanol/water, $1: 99, v / v$, containing $1 \mathrm{mmol} / \mathrm{L}$ formic acid) and solvent $\mathrm{B}$ (methanol/water, 99:1, $v / v$, containing $1 \mathrm{mmol} / \mathrm{L}$ formic acid). The mobile phase was delivered at $0.3 \mathrm{~mL} / \mathrm{min}$. A gradient program was used as follows: $0-2 \mathrm{~min}$, at $2 \%$ solvent $\mathrm{B}$; 2-32 min, from $2 \%$ to $98 \%$ solvent B; $32-37 \mathrm{~min}$, at $98 \%$ solvent $\mathrm{B}$; and $37-42 \mathrm{~min}$, at $2 \%$ solvent $\mathrm{B}$. To support the metabolite profiling, Pallas MetabolExpert software (Pallas 3.7; CompuDrug International, Sedona, AZ, USA) was used to obtain prior knowledge of the likely metabolic pathways of Chishao monoterpene glycosides. For the profiling of Chishao monoterpene glycosides present in samples of XueBijing injection, $50 \%$ methanol was used to dilute the samples before analysis; for the profiling of monoterpene glycosides in samples of Chishao, 50\% methanol was used to extract the samples and the extract was centrifuged to yield the supernatant, which was diluted with 50\% methanol before analysis. Plasma samples from the same time point were pooled for the profiling 
of unchanged and metabolized Chishao monoterpene glycosides in human and rat samples, whereas excretory samples of the same type from the same time period were also pooled. Sample preparation was performed using methanol as a precipitant with a volumetric precipitant-to-sample ratio of 3:1. After centrifugation, the supernatant was concentrated, under reduced pressure, for analysis.

In addition, an AB Sciex API 4000 Q Trap mass spectrometer (Toronto, Canada), interfaced via a Turbo $\mathrm{V}$ ion source with a Waters Acquity UPLC separation module, was used to measure concentrations of Chishao monoterpene glycosides in human and rat samples, as well as in vitro biological samples. The mass spectrometer parameters were optimized to maximize generation of the singly deprotonated species and to produce the characteristic product ions of the test compounds. The precursor-to-product ion pairs used for multiple-reaction monitoring of paeoniflorin, oxypaeoniflorin, and albiflorin were $m / z 525 \rightarrow 449,525 \rightarrow 479$, and $495 \rightarrow 137$, respectively. Chromatographic separation was achieved on a Shiseido Capcell Pak C18 3.0- $\mu \mathrm{m}$ column ( $50 \mathrm{~mm} \times 2.0 \mathrm{~mm}$ id; Tokyo, Japan). The mobile phase was the same as that used for the preceding profiling assays and was also delivered at $0.3 \mathrm{~mL} / \mathrm{min}$. A pulse gradient chromatographic method was used, which was modified from the method by Li et al ${ }^{[49]}$. The gradient program was as follows: $0-0.5 \mathrm{~min}$, at $2 \%$ solvent $\mathrm{B} ; 0.5-3 \mathrm{~min}$, at $55 \%$ solvent B; and 3-5 min, at $2 \%$ solvent B. Sample preparation was performed using methanol as a precipitant with a volumetric precipitant-to-sample ratio of 3:1. After centrifugation, the supernatants were applied to liquid chromatography/tandem mass spectrometry. Matrix-matched calibration curves (37-9000 nmol/L for quantification of paeoniflorin and oxypaeoniflorin or 111-9000 nmol/L for quantification of albiflorin) were constructed using weighted $(1 / X)$ linear regression of the peak area $(Y)$ against the corresponding nominal analyte concentration $(X, \mathrm{nmol} / \mathrm{L})$. Assay validation was performed according to the US FDA guidance on bioanalytical validation (2013) to prove that the bioanalytical assay was reliable for the intended application. The lower limits of quantification were 37, 37, and $111 \mathrm{nmol} / \mathrm{L}$ for measurement of paeoniflorin, oxypaeoniflorin, and albiflorin in plasma samples, respectively; the assay precision was $<20 \%$, whereas the assay accuracy was $80 \%-120 \%$.

\section{Data processing}

Plasma PK parameters of Chishao monoterpene glycosides were determined using non-compartmental analysis with Kinetica software (version 5.0; Thermo Scientific, Philadelphia, PA, USA). The area under the concentration-time curve up to the last measured point in time $\left(\mathrm{AUC}_{0-\mathrm{t}}\right)$ was calculated using the trapezoidal rule. The $\mathrm{AUC}_{0-\infty}$ was generated by extrapolating $\mathrm{AUC}_{0-\mathrm{t}}$ to infinity using the elimination rate constant $k_{\mathrm{e}}$ and the last measured concentration $\left(C_{\mathrm{t}}\right)$. The total plasma clearance $\left(\mathrm{CL}_{\mathrm{tot}, \mathrm{p}}\right)$ was estimated by dividing the dose by the $\mathrm{AUC}_{0-\infty}$, and the distribution volume at steady state $\left(V_{\mathrm{SS}}\right)$ was estimated by multiplying the $\mathrm{CL}_{\mathrm{tot}, \mathrm{p}}$ by the mean residence time (MRT). The $t_{1 / 2}$ was calculated using the relationship
$0.693 / k_{\mathrm{e}}$. For infusion at a constant rate, the plasma concentration at steady state $\left(C_{\mathrm{SS}}\right)$ and the amount in the body at steady state $\left(A_{\mathrm{SS}}\right)$ were estimated using the following equations:

$$
\begin{aligned}
& C_{\mathrm{SS}}=R_{0} / C_{\mathrm{tot}, \mathrm{p}} \\
& A_{\mathrm{SS}}=R_{0} / k_{\mathrm{e}}
\end{aligned}
$$

where $R_{0}$ is the infusion rate with respect to paeoniflorin (5) in $\mathrm{mmol} / \mathrm{h}$. Dose proportionality was assessed using the regression of log-transformed data (the Power Model), and the criteria was calculated according to a method by Smith et $a l^{[50]}$. The correlation coefficient $\left(r^{2}\right)$, slope, and $90 \%$ confidence intervals for the slope were calculated, and inference of linear pharmacokinetics was made based on a theoretical slope of one and a confidence limit of 0.84 to 1.16 . The accumulation ratio $\left(R_{\mathrm{ac}}\right)$ was calculated to indicate the extent of accumulation during multiple doses of XueBijing injection using the following equations:

$$
R_{\mathrm{ac}}=\mathrm{AUC}_{0-\infty(\text { Day-7) }} / \mathrm{AUC}_{0-\infty(\text { Day }-1)}
$$

The renal excretory clearance $\left(\mathrm{CL}_{\mathrm{R}}\right)$ and the hepatobiliary excretory clearance $\left(\mathrm{CL}_{\mathrm{B}}\right)$ were estimated by dividing the cumulative amount excreted into urine $\left(\right.$ Cum. $\left.A_{\mathrm{e}-\mathrm{U}}\right)$ and into bile (Cum. $\left.A_{\text {e- } \mathrm{B}}\right)$, respectively, by the plasma $\mathrm{AUC}_{0-\infty}$. The fractions of dose excreted into urine $\left(f_{\mathrm{e}-\mathrm{U}}\right)$ and into bile $\left(f_{\mathrm{e}-\mathrm{B}}\right)$ were established using the relationships Cum.A $A_{\mathrm{e}-\mathrm{U}} /$ Dose and Cum. $A_{\mathrm{e}-\mathrm{B}} /$ Dose, respectively.

All data are expressed as the mean \pm standard deviation. Statistical analysis was performed using IBM SPSS Statistics software (version 19.0; IBM, Somers, NY, USA). A $P$ value $<0.05$ was considered statistically significant.

\section{Results}

Chishao monoterpene glycosides present in XueBiJing injection

Two literature references provide the most comprehensive information on the chemical constituents present in P lactiflora

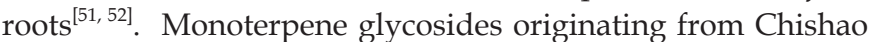
could be important for XueBijing therapy. As shown in Figure 1 and Table 1 , a total of 18 Chishao monoterpene glycosides were detected in the samples of eight lots of XueBijing injection. Paeoniflorin (5) exhibited the highest content level $(2.24-2.72 \mathrm{mmol} / \mathrm{L})$ in the herbal injection, and its $\log P$ value was -0.91 . Oxypaeoniflorin $(6 ; \log P,-1.47)$ and albiflorin (4; $-0.6)$ were also present, although at significantly lower levels (0.10-0.12 and $0.08-0.16 \mathrm{mmol} / \mathrm{L}$, respectively). The other detected monoterpene glycosides (Figure 1A; $\log P,-2.24-$ $0.69)$, including benzoylpaeoniflorin (11), galloylpaeoniflorin (15), desbenzoylpaeoniflorin (3), mudanpioside E (9), isomer of galloylpaeoniflorin or galloylalbiflorin (16), mudanpioside F (1), ortho-oxypaeoniflorin (8), isomer of oxypaeoniflorin (7), galloyloxypaeoniflorin (18), isomer of galloylpaeoniflorin or galloylalbiflorin (17), mudanpioside J (14), benzoyloxypaeoniflorin (12), mudanpioside C (13), 1-O- $\beta$ - $D$-glucopyranosylpaeonisuffrone (2), and 6'-O-galloyl desbenzoylpaeoniflorin (10), had even lower content levels ranging from 0.001 to 0.06 $\mathrm{mmol} / \mathrm{L}$. The chemical structures of 5, 6, and 4 are shown in Supplemental Information Appendix S1. XueBijing injection exhibited small lot-to-lot variability with regard to the content levels of $\mathbf{5}$ and $\mathbf{6}$, indicated by relative standard deviations 
A (Chromatogram of XueBiJing injection)

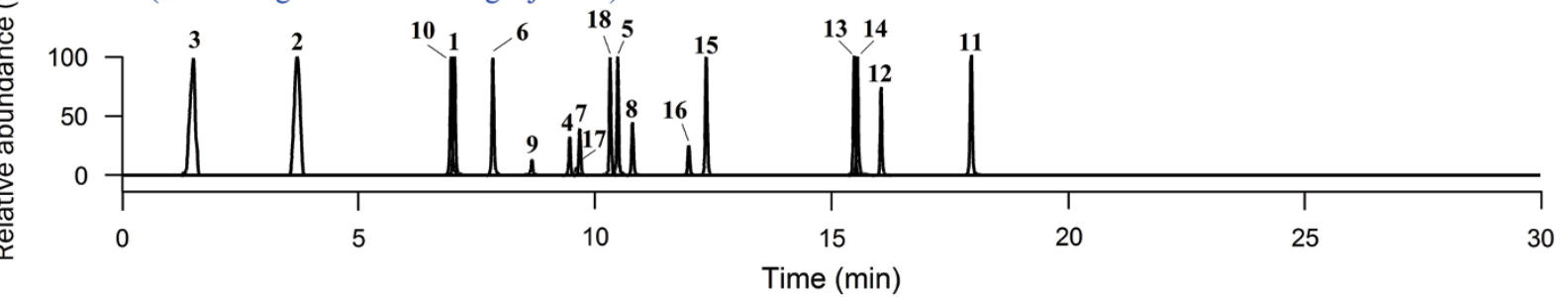

B (Mean content levels of monoterpene glycosides in different lots of Chishao)

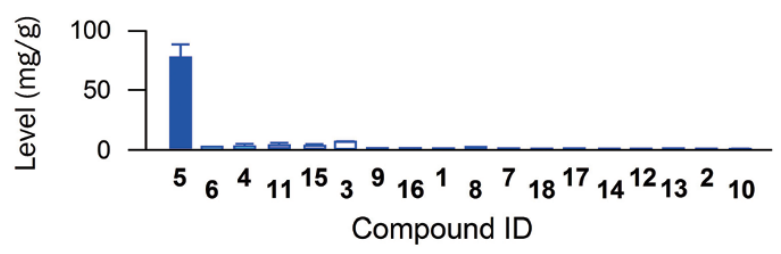

D (Doses of Chishao monoterpene glycosides from XueBiJing injection)

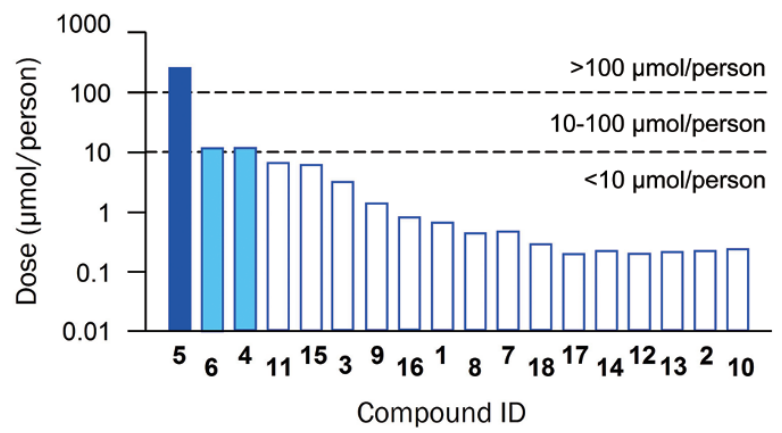

C (Mean content levels of Chishao monoterpene glycosides in different lots of XueBiJing injection)

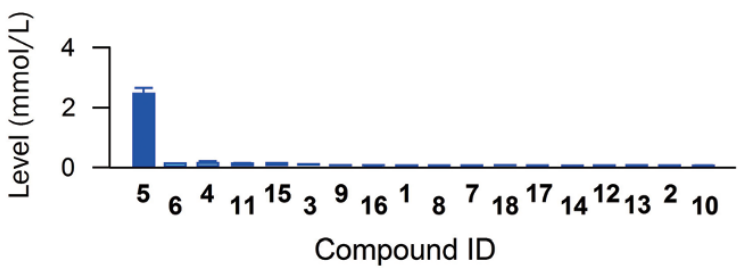

E (Percentage doses of Chishao monoterpene glycosides in the total dose of the monoterpene glycosides in XueBiJing injection)

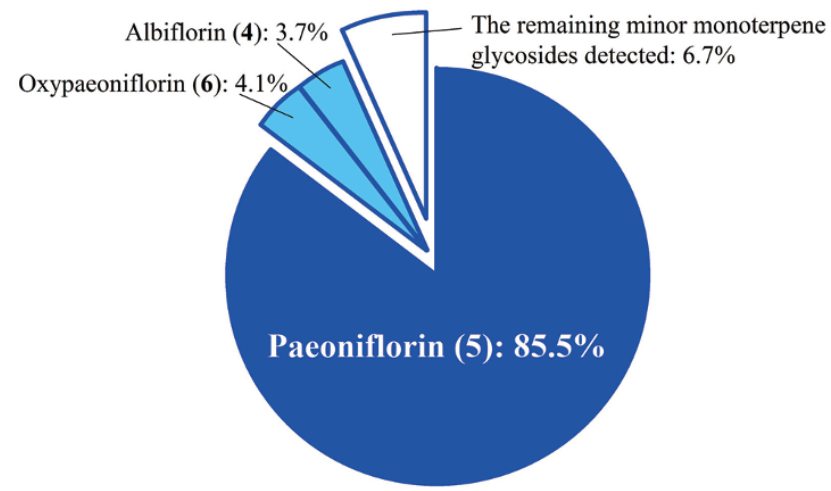

Figure 1. LC/TOF-MS -based detection and measurement of Chishao monoterpene glycosides present in XueBiJing injection. (A) Chromatogram of XueBiJing injection showing the detection of Chishao monoterpene glycosides; (B) mean content levels of monoterpene glycosides in the raw materials of Chishao (P lactiflora roots); (C) mean content levels of monoterpene glycosides in different lots of XueBiJing injection (lot numbers: 1309271, 1309281, 1309291, 1309301, 1405301, 1406161, 1408191, and 1410081); (D) clinical daily doses of monoterpene glycosides from XueBiJing injection (1309301); (E) percentage doses of monoterpene glycosides in the total dose of monoterpene glycosides in XueBiJing injection (1309301). The chemical names and detection information of monoterpene glycosides (shown as compound ID) are listed in Table 1.

of 5.8 and $6.8 \%$, respectively. Relative standard deviation values for 4 and the remaining minor Chishao monoterpene glycosides appeared to be greater, $i e, 27.0 \%$ and $11.7 \%-40.2 \%$, respectively.

XueBijing injection (lot number, 1309301) was used in the current study. The content levels of paeoniflorin (5), oxypaeoniflorin (6), and albiflorin (4) were 2.38, 0.11, and $0.10 \mathrm{mmol} / \mathrm{L}$, respectively, and those of the remaining Chishao monoterpene glycosides were $0.002-0.06 \mathrm{mmol} / \mathrm{L}$. According to their dose levels from XueBijing injection $(100 \mathrm{~mL} /$ person), these compounds could be divided into the following three classes: $>100 \mu \mathrm{mol} /$ person $(5 ; 238 \mu \mathrm{moL} /$ person), 10-100 $\mu \mathrm{mol} /$ person $(\mathbf{6} ; 11.3 \mu \mathrm{mol} /$ person and $4 ; 10.2 \mu \mathrm{mol} /$ person), and $<10 \mu \mathrm{mol} /$ person (the remaining minor Chishao monoter- pene glycosides; 0.19-6.15 $\mu \mathrm{mol} /$ person; Figure 1D). The dose level of $\mathbf{5}$ was $85.5 \%$ of the total dose of Chishao monoterpene glycosides present in the injection (Figure 1E). The dose levels of 6 and 4 together accounted for $7.8 \%$ of the total dose of Chishao monoterpene glycosides present in the injection. The dose levels of the remaining 15 Chishao monoterpene glycosides together accounted for only $6.7 \%$ of the total dose of Chishao monoterpene glycosides present in the injection.

Chishao monoterpene glycosides detected in plasma and excretory samples of human subjects and rats receiving an intravenous infusion of XueBiJing injection

A total of 11 Chishao monoterpene glycosides were detected in plasma samples of human subjects receiving a single 
Table 1. Detection of Chishao monoterpene glycosides present in XueBiJing injection.

\begin{tabular}{|c|c|c|c|c|c|c|}
\hline \multirow[b]{2}{*}{ ID } & \multirow{2}{*}{ Compound } & \multicolumn{3}{|c|}{ LC/TOF-MS ${ }^{\mathrm{E}}$ data } & \multirow{2}{*}{$\begin{array}{l}\text { Molecular } \\
\text { mass } \\
\text { (Da) }\end{array}$} & \multirow{2}{*}{$\begin{array}{c}\text { Molecular } \\
\text { formula }\end{array}$} \\
\hline & & $\begin{array}{c}t_{R} \\
(\mathrm{~min})\end{array}$ & $\frac{[\mathrm{M}-\mathrm{H}]^{-}}{(\mathrm{m} / \mathrm{z})}$ & $\begin{array}{l}\text { Fragmentation profile } \\
\qquad(\mathrm{m} / \mathrm{z})\end{array}$ & & \\
\hline 1 & Mudanpioside F & 7.03 & 343.1393 & $109.0654,151.0760,163.0755,181.0865$ & 344.1471 & $\mathrm{C}_{16} \mathrm{H}_{24} \mathrm{O}_{8}$ \\
\hline 2 & 1-O-B-D-Glucopyranosyl-Paeonisuffrone & 3.71 & 359.1342 & $179.0703,351.1307,197.0805,217.1185$ & 360.1420 & $\mathrm{C}_{16} \mathrm{H}_{24} \mathrm{O}_{9}$ \\
\hline 3 & Desbenzoylpaeoniflorin & 1.53 & 375.1291 & $165.0549,195.0660,345.1184$ & 376.1369 & $\mathrm{C}_{16} \mathrm{H}_{24} \mathrm{O}_{10}$ \\
\hline 4 & Albiflorin & 9.46 & 479.1553 & $121.0290,165.0552,375.1330,479.1554$ & 480.1632 & $\mathrm{C}_{23} \mathrm{H}_{28} \mathrm{O}_{11}$ \\
\hline 5 & Paeoniflorin & 10.47 & 479.1553 & $121.0291,165.0553,327.1078,449.1446$ & 480.1632 & $\mathrm{C}_{23} \mathrm{H}_{28} \mathrm{O}_{11}$ \\
\hline 6 & Oxypaeoniflorin & 7.85 & 495.1503 & $93.0341,137.0237,465.1395$ & 496.1581 & $\mathrm{C}_{23} \mathrm{H}_{28} \mathrm{O}_{12}$ \\
\hline 7 & Oxypaeoniflorin isomer & 9.68 & 495.1503 & 287.0926, 361.1495, 379.1601 & 496.1581 & $\mathrm{C}_{23} \mathrm{H}_{28} \mathrm{O}_{12}$ \\
\hline 8 & Ortho-oxypaeoniflorin & 10.80 & 495.1503 & 137.0244 & 496.1581 & $\mathrm{C}_{23} \mathrm{H}_{28} \mathrm{O}_{12}$ \\
\hline 9 & Mudanpioside E & 8.67 & 525.1608 & $351.1286,385.1134,417.1394$ & 526.1686 & $\mathrm{C}_{24} \mathrm{H}_{30} \mathrm{O}_{13}$ \\
\hline 10 & 6'-O-Galloyl-desbenzoylpaeoniflorin & 6.96 & 527.1401 & $479.1188,497.1299$ & 528.1479 & $\mathrm{C}_{23} \mathrm{H}_{28} \mathrm{O}_{14}$ \\
\hline 11 & Benzoylpaeoniflorin & 17.95 & 583.1816 & 121.0292, 165.0557, 431.1342, 553.1700 & 584.1894 & $\mathrm{C}_{30} \mathrm{H}_{32} \mathrm{O}_{12}$ \\
\hline 12 & Benzoyloxypaeoniflorin & 16.05 & 599.1765 & $137.0240,477.1389$ & 600.1843 & $\mathrm{C}_{30} \mathrm{H}_{32} \mathrm{O}_{13}$ \\
\hline 13 & Mudanpioside C & 15.53 & 599.1765 & 281.0662, 431.1335, 477.1391, 581.1655 & 600.1843 & $\mathrm{C}_{30} \mathrm{H}_{32} \mathrm{O}_{13}$ \\
\hline 14 & Mudanpioside J & 15.56 & 629.1870 & 281.0662, 431.1335, 477.1391, 581.1655 & 630.1949 & $\mathrm{C}_{31} \mathrm{H}_{34} \mathrm{O}_{14}$ \\
\hline 15 & Galloylpaeoniflorin & 12.36 & 631.1663 & $271.0452,313.0561,491.1188,613.1550$ & 632.1741 & $\mathrm{C}_{30} \mathrm{H}_{32} \mathrm{O}_{15}$ \\
\hline 16 & Isomer of galloylpaeoniflorin or galloylalbiflorin & 11.97 & 631.1663 & $179.0707,465.1401$ & 632.1741 & $\mathrm{C}_{30} \mathrm{H}_{32} \mathrm{O}_{15}$ \\
\hline 17 & Isomer of galloylpaeoniflorin or galloylalbiflorin & 9.61 & 631.1663 & $165.0548,313.0579,401.1445,503.1752$ & 632.1741 & $\mathrm{C}_{30} \mathrm{H}_{32} \mathrm{O}_{15}$ \\
\hline 18 & Galloyloxypaeoniflorin & 10.32 & 647.1612 & 433.1494, 605.2576 & 648.1690 & $\mathrm{C}_{30} \mathrm{H}_{32} \mathrm{O}_{16}$ \\
\hline
\end{tabular}

$t_{\mathrm{R}}$, chromatographic retention time; Number in bold, product ion of base peak.

75-min intravenous infusion of $100 \mathrm{~mL}$ XueBijing injection (Figure 2A). These herbal compounds were not detected in the plasma samples before dosing. The levels of systemic exposure to the monoterpene glycosides depended mainly on the individual compound daily dose levels from the herbal injection. This was indicated by paeoniflorin (5), which was the only monoterpene glycoside detected in considerable amount in the plasma samples after dosing started. The other monoterpene glycosides detected in plasma included oxypaeoniflorin (6) and albiflorin (4), but they had significantly lower levels of systemic exposure than 5. Although benzoylpaeoniflorin (11), galloylpaeoniflorin (15), mudanpioside E (9), isomer of galloylpaeoniflorin or galloylalbiflorin (16), mudanpioside F (1), mudanpioside J (14), benzoyloxypaeoniflorin (12), and mudanpioside C (13) were also detected in human plasma after dosing, they were found at very low levels. In the human urine samples collected after dosing intravenous XueBijing injection, there were 18 unchanged Chishao monoterpene glycosides that were detected (Figure 2B). As for the plasma samples, 5 was also the most substantially detected monoterpene glycoside in urine, whereas urinary 6, 4, 11, 15, desbenzoylpaeoniflorin (3), 9, 16, 1, ortho-oxypaeoniflorin (8), isomer of oxypaeoniflorin (7), galloyloxypaeoniflorin (18), isomer of galloylpaeoniflorin or galloylalbiflorin (17), 14, 12, 13, $1-O-\beta$ - $D$-glucopyranosyl-paeonisuffrone (2), and 6'-O-galloyl desbenzoylpaeoniflorin (10) were found at low levels. The amounts of Chishao monoterpene glycosides excreted into urine, like their levels of systemic exposure, also depended on their individual daily dose levels from XueBijing injection.

Metabolite profiling was further performed for paeoniflorin (5). The reported metabolites (paeonimetabolin I, paeonime- tabolin II, and paeoniflorgenin) of oral paeoniflorin, by the intestinal microflora ${ }^{[41-44]}$, were not detected in the plasma or urine samples of the human subjects receiving intravenous XueBijing injection. Other predicted metabolites of 5, including the acetylated, hydroxylated, alcoholic-OH-reduced, glucuronidated metabolites, and the further metabolites, were also not detected in the human samples. In addition, paeoniflorin could not be transformed into oxypaeoniflorin by incubation with human liver microsomes fortified with reduced $\beta$-nicotinamide adenine dinucleotide phosphate (data not shown).

In rats, a total of 11 Chishao monoterpene glycosides were detected in plasma samples during and after a single 30-min intravenous infusion of XueBijing injection at $10 \mathrm{~mL} / \mathrm{kg}$ (Figure 2C). As for human subjects, paeoniflorin (5) was the most abundant monoterpene glycoside detected in plasma samples of rats receiving the herbal injection. The other monoterpene glycosides, including oxypaeoniflorin (6), albiflorin (4), benzoylpaeoniflorin (11), mudanpioside E (9), mudanpioside $\mathrm{F}$ (1), ortho-oxypaeoniflorin (8), galloyloxypaeoniflorin (18), benzoyloxypaeoniflorin (12), mudanpioside $\mathrm{C}(\mathbf{1 3})$, and 1-O- $\beta$ D-glucopyranosyl-paeonisuffrone (2), were also detected in plasma, but at quite low levels. In rat urine, 15 Chishao monoterpene glycosides were detected during and after dosing XueBijing injection; the amounts excreted depended on their compound doses (Figure 2D). Hepatobiliary excretion of these monoterpene glycosides (Figure 2E) was slower than renal excretion. No significant metabolite of $\mathbf{5}$ was detected in the rat samples. The fecal excretion of Chishao monoterpene glycosides was negligible, and the amounts of the compounds excreted into feces never exceeded the amounts excreted into bile. 

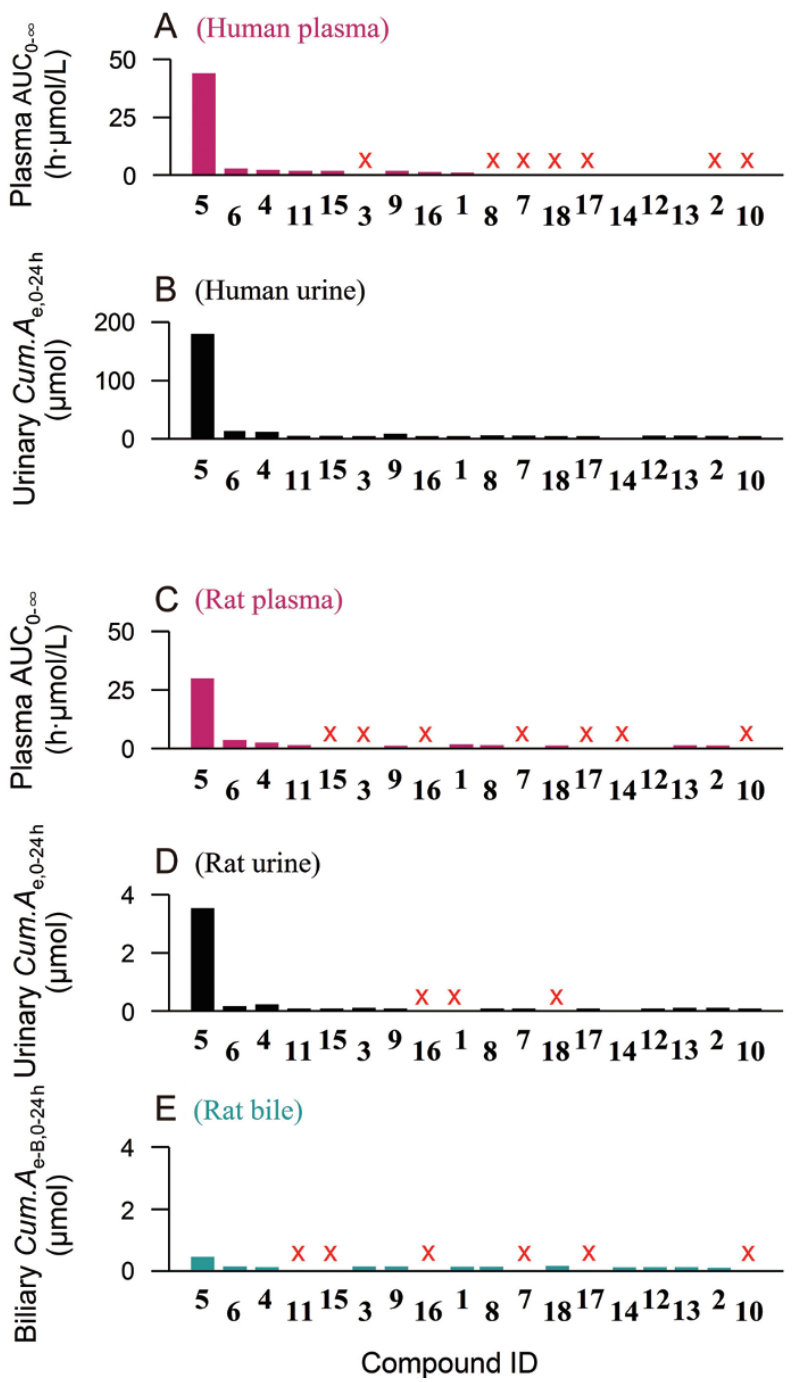

Figure 2. Systemic exposure to and excretion of Chishao monoterpene glycosides in human subjects $(A-B)$ and rats $(C-E)$ intravenously receiving XueBiJing injection. The chemical names of monoterpene glycosides (shown as compound ID) are listed in Table 1. The symbol " $x$ " (in red) denotes the monoterpene glycoside that was not detected. The human subjects received a 75-min intravenous infusion of 100-mL XueBiJing injection (diluted in $100 \mathrm{~mL}$ of $0.9 \% \mathrm{NaCl}$ injection). The rats received a 30-min intravenous infusion of the injection at $10 \mathrm{~mL} / \mathrm{kg}$. Because of the sample pooling, no standard deviation is shown.

\section{Membrane permeability and plasma protein binding of Chishao} monoterpene glycosides

The purified compounds paeoniflorin, oxypaeoniflorin, and albiflorin exhibited poor membrane permeability across Caco- 2 cell monolayer. This was indicated by their $P_{\text {app,A-B }}$ values, which were $5.2 \times 10^{-7}, 5.8 \times 10^{-7}$, and $3.5 \times 10^{-7} \mathrm{~cm} / \mathrm{s}$. The transports were based on passive diffusion with efflux ratios of 1.1-1.4. Paeoniflorin, oxypaeoniflorin, and albiflorin were poorly bound to human plasma protein with concentrationindependent $f_{\mathrm{u}}$ values of $82.3 \%, 80.2 \%$, and $82.6 \%$, respectively, and to rat plasma protein with concentration-independent $f_{\mathrm{u}}$ values of $93.8 \%, 89.5 \%$, and $81.2 \%$, respectively.
Plasma pharmacokinetics and renal excretion of paeoniflorin in human subjects receiving single and multiple intravenous infusions of XueBiJing injection

No serious adverse event was observed during the period of human study. After a single intravenous infusion of XueBiJing injection (ie, 75-min infusion of $100 \mathrm{~mL}$ of XueBijing injection plus $100 \mathrm{~mL}$ of the $\mathrm{NaCl}$ injection, 150-min infusion of 100 $\mathrm{mL}$ of XueBijing injection plus $200 \mathrm{~mL}$ of the $\mathrm{NaCl}$ injection, or 75-min infusion of $50 \mathrm{~mL}$ of XueBijing injection plus 100 $\mathrm{mL}$ of the $\mathrm{NaCl}$ injection), the liver and kidney function indicators for all the human subjects were within normal ranges. During the following multiple dose treatment with the herbal injection, two male subjects displayed abnormalities in liver or kidney function; one subject had transient elevation of ALT (up to $73 \mathrm{IU} / \mathrm{L}$; the normal ALT range is $0-40 \mathrm{IU} / \mathrm{L}$ ) and AST (up to $45 \mathrm{IU} / \mathrm{L}$; the normal AST range is $0-40 \mathrm{IU} / \mathrm{L}$ ) on $\mathrm{d} 7$ after initiating the multiple dose treatment, and the other had transient elevation of ALT (up to $50 \mathrm{IU} / \mathrm{L}$ ) also on d 7. These two human subjects were found to have normal values within $7 \mathrm{~d}$ after study completion.

Figure 3 shows the plasma concentrations of paeoniflorin (5) over time, during and after an intravenous infusion and multiple infusions of XueBiJing injection in human subjects. Table 2 summarizes the human plasma and urinary PK parameters of 5 during and after an intravenous infusion of the injection. The plasma concentration of 5 rose as the infusion was maintained, and the plasma $C_{\max }$ was measured at the time just prior to completion of the infusion, ie, $75 \mathrm{~min}(1.25 \mathrm{~h})$ after the initiation of infusion for groups A and C and $150 \mathrm{~min}$ (2.5 h) for group B. After the infusion was stopped, the plasma concentration of 5 declined, with a mean $t_{1 / 2}$ of $1.11 \pm 0.03 \mathrm{~h}$ for the three groups. This indicates that XueBiJing injection was infused for only 1.1 (for groups A and C) and $2.3 t_{1 / 2}$ of 5 (for group $\mathrm{B})$, which were shorter than the $3.3 t_{1 / 2}$ required to reach $90 \%$ of the plateau. In group $B$, both the mean plasma $C_{\max }$ and mean plasma $\mathrm{AUC}_{0-\infty}$ of $\mathbf{5}$ were significantly greater among female subjects than among male subjects $(P<0.01)$. However, when the dose was corrected for body weight, the gender differences were insignificant in these systemic exposure data $(P=0.3)$. Unlike in group $B$, the female subjects in groups $A$ and $C$ exhibited lower mean plasma $C_{\max }$ than the male subjects $(P<0.05)$, and the female subjects in group $C$ also had lower mean plasma $\mathrm{AUC}_{0-\infty}$ than the male subjects $(P=0.01)$ when the dose was corrected for body weight. Among the three groups receiving different dosage regimens, there was no significant difference in $V_{\mathrm{SS}}(P=0.06-0.9)$ or $\mathrm{CL}_{\text {tot,p }}(P=0.7-$ $0.8)$ (Table 2). The mean $V_{\mathrm{SS}}$ values of $5(0.19 \pm 0.03 \mathrm{~L} / \mathrm{kg}$; for all groups) was larger than human plasma volume $(0.04 \mathrm{~L} / \mathrm{kg})$ but smaller than human extracellular volume $(0.26 \mathrm{~L} / \mathrm{kg})$, suggesting that this monoterpene glycoside had a small volume of distribution and was predominantly restricted to the extracellular fluid. Circulating 5 had low clearance, as indicated by its mean $\mathrm{CL}_{\text {tot, }}\left(0.12 \pm 0.02 \mathrm{~L} \cdot \mathrm{h}^{-1} \cdot \mathrm{kg}^{-1}\right.$ for all groups), which was only $5 \%$ of human cardiac plasma output $\left(2.4 \mathrm{~L} \cdot \mathrm{h}^{-1} \cdot \mathrm{kg}^{-1}\right)^{[53]}$. There were no significant gender differences in $t_{1 / 2}, V_{\mathrm{SS}}$, or $\mathrm{CL}_{\text {tot, } \mathrm{p}}$ $(P=0.1-0.9)$, with the exception that female subjects in group $C$ 
had larger mean $V_{\mathrm{SS}}$ and $\mathrm{CL}_{\mathrm{tot}, \mathrm{p}}$ values than the male subjects from the same group $(P<0.01)$. The mean $C_{\mathrm{SS}}$ values of 5 were estimated to be 29.1, 14.6, and $14.2 \mu \mathrm{mol} / \mathrm{L}$ for groups A, B, and $\mathrm{C}$, respectively; the mean $A_{\mathrm{SS}}$ values were estimated to be 299, 155, and $152 \mu \mathrm{mol}$, respectively. The interindividual variances in $C_{\mathrm{SS}}$ were $15.4 \%-28.3 \%$, whereas those of $A_{\mathrm{SS}}$ were $8.5 \%-12.4 \%$. During multiple infusions of XueBijing injection, the accumulation of $\mathbf{5}$ in the plasma was negligible (Figure 3). The mean $\mathrm{AUC}_{0-\infty}$ on $\mathrm{d} 7 \mathrm{did}$ not differ significantly from that on $\mathrm{d} 1(P=0.06)$, and the $R_{\mathrm{ac}}$ was 0.85 . The mean $V_{\mathrm{SS}}$ and mean $\mathrm{CL}_{\text {tot,p }}$ on $\mathrm{d} 7 \mathrm{did}$ not differ significantly from those on $\mathrm{d} 1(\mathrm{P}=$ 0.1 and 0.2 , respectively). The plasma PK parameters of 5 on $\mathrm{d}$ 7 during and after multiple intravenous infusions of the injection in six male subjects are shown in Supplementary Information Table S1.

Renal excretion was found to be the major elimination pathway of paeoniflorin (5). This was indicated by the $C L_{R}$ of 5 , which accounted for $58.3 \%-63.6 \%$ of the $\mathrm{CL}_{\text {tot,p }}$. The $\mathrm{CL}_{\mathrm{R}}$ value was 0.7-0.9 times as much as the product of the reported human GFR $\left(0.11 \mathrm{~L} \cdot \mathrm{h}^{-1} \cdot \mathrm{kg}^{-1}\right)^{[54]}$ and the $f_{\mathrm{u}}$ of $5(82.3 \%)$; this suggests that the renal excretion of 5 is mainly based on glomerular filtration. No significant gender difference in

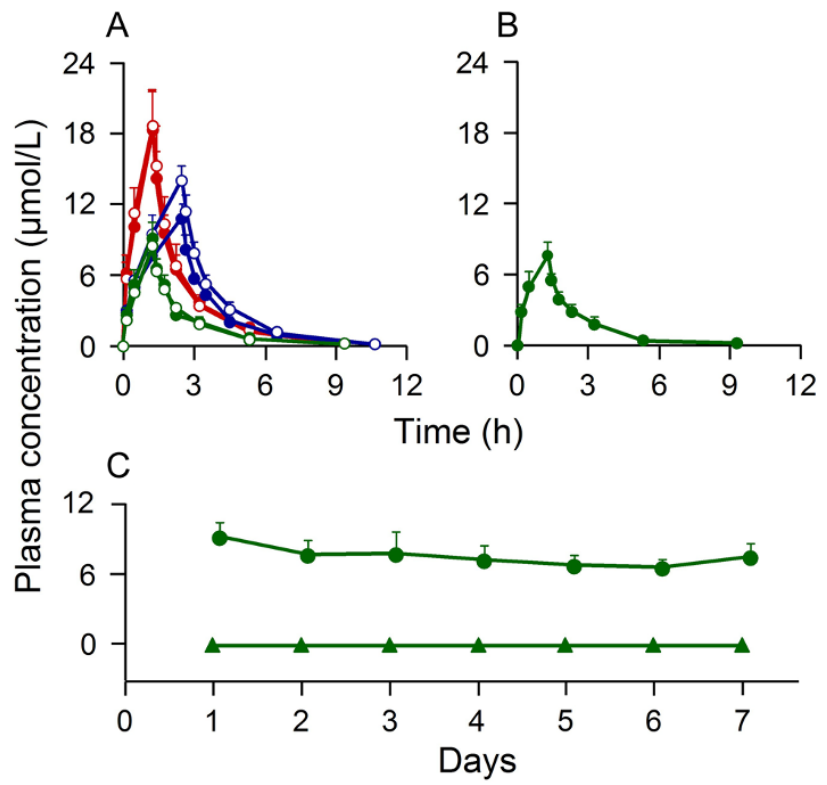

Figure 3. Mean plasma concentrations of paeoniflorin (5) over time in male (solid circles) and female human subjects (open circles) receiving an intravenous infusion of XueBiJing injection (A). Red lines: the subjects receiving a 75 -min intravenous infusion of $100-\mathrm{mL}$ XueBiJing injection (diluted in $100 \mathrm{~mL}$ of $0.9 \% \mathrm{NaCl}$ injection); blue lines: the subjects receiving a 150-min infusion of 100-mL XueBiJing injection (diluted in $200 \mathrm{~mL}$ of $0.9 \% \mathrm{NaCl}$ injection); green lines: the subjects receiving a 75min infusion of $50-\mathrm{mL}$ XueBiJing injection (diluted in $100 \mathrm{~mL}$ of $0.9 \%$ $\mathrm{NaCl}$ injection). In addition, six of the male subjects continued to receive the same dose of the injection (green line) each day for the following six days; panel (B): mean plasma concentrations of 5 over time on d 7; panel (C): mean daily plasma concentrations of 5 at 0 (solid triangles) and 75 min (solid circles) after daily dosing from $d 1$ to $d 7$. Each milliliter of the injection contained $1.14 \mathrm{mg}$ of 5 .
Cum. $A_{\mathrm{e}-\mathrm{U}}$ of $\mathbf{5}$ was observed in the human subjects for each of the three groups receiving XueBiJing injection, regardless of whether the doses were corrected for body weight $(P=0.2-0.5)$. Although no significant gender difference in $\mathrm{CL}_{R}$ of 5 was observed in group B, the female subjects from groups A and $C$ exhibited greater mean $\mathrm{CL}_{\mathrm{R}}$ than the male subjects from the same groups $(P<0.03)$. No significant gender difference in $f_{\mathrm{e}-\mathrm{U}}$ of 5 was observed in the human subjects $(P=0.2-0.8)$.

Plasma pharmacokinetics, tissue distribution, and excretion of paeoniflorin in rats receiving a single intravenous dose of XueBiJing injection

Table 3 summarizes the rat plasma PK parameters of paeoniflorin (5) during and after an intravenous infusion of XueBiJing injection at 5, 10, and $20 \mathrm{~mL} / \mathrm{kg}$; Figure 4 shows the plasma concentrations of 5 over time. As in the human subjects, 5 resided mainly in the extracellular fluid, with a mean $V_{\text {SS }}$ of $0.28 \pm 0.03 \mathrm{~L} / \mathrm{kg}$ (for all dose levels), which was between rat plasma volume $(0.03 \mathrm{~L} / \mathrm{kg})$ and rat extracellular volume $(0.30 \mathrm{~L} / \mathrm{kg})$. Clearance of 5 in rats was not high, as indicated by the mean $\mathrm{CL}_{\text {tot,p }}\left(0.88 \pm 0.03 \mathrm{~L} \cdot \mathrm{h}^{-1} \cdot \mathrm{kg}^{-1}\right.$ for all dose levels), which was $12 \%$ of rat cardiac plasma output $\left(7.32 \mathrm{~L} \cdot \mathrm{h}^{-1} \cdot \mathrm{kg}^{-1}\right)$. The mean $t_{1 / 2}$ of 5 in the rats was short, ie, $0.42 \pm 0.05 \mathrm{~h}$ (for all dose levels). As shown in Table 4, renal excretion was the major elimination pathway of 5 in rats, with a $f_{\mathrm{e}-\mathrm{U}}$ of $50.2 \%$. The $\mathrm{CL}_{\mathrm{R}} /\left(\mathrm{GFR} \times f_{\mathrm{u}}\right)$ ratio of 5 in the rats was 1.4 , suggesting the involvement of a glomerular-filtration-based mechanism. Taken together, rats exhibited similar PK features of intravenous 5 to those of humans. Accordingly, multiple types of rat studies, which were difficult to perform in human subjects for ethical reasons, were conducted to help better understand the pharmacokinetics and dispositions of 5 resulting from intravenously dosed XueBiJing injection.

In rats, levels of systemic exposure to paeoniflorin (5) with respect to plasma $C_{\max }$ and $\mathrm{AUC}_{0-\infty}$ increased proportionally as the dose of XueBijing injection increased from 5 to $20 \mathrm{~mL} / \mathrm{kg}$ (Table 5). Unlike conventional synthetic drug injection, herbal

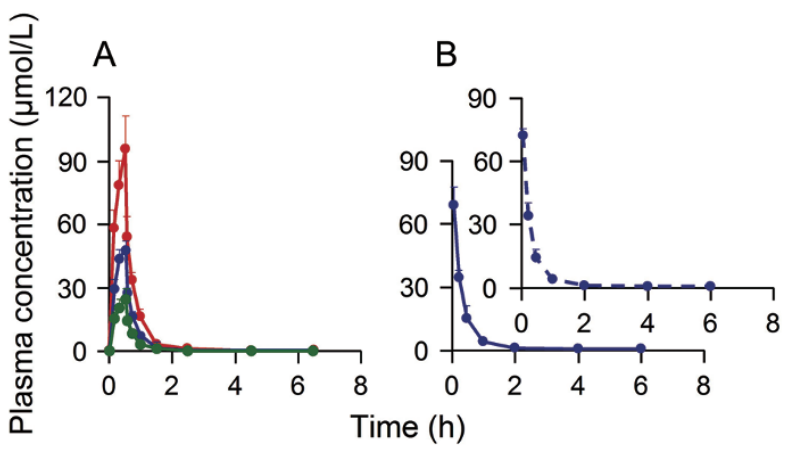

Figure 4. Mean plasma concentrations of paeoniflorin (5) over time in rats receiving a 30-min intravenous infusion of XueBiJing injection at 5 (green line), 10 (blue line), and $20 \mathrm{~mL} / \mathrm{kg}$ (red line) (A) and an intravenous bolus dose of XueBiJing injection at $10 \mathrm{~mL} / \mathrm{kg}$ (blue solid line) and purified paeoniflorin at $11.4 \mathrm{mg} / \mathrm{mL}$ (blue dashed line) (B). Each milliliter of the injection contained $1.14 \mathrm{mg}$ of 5 . 
Table 2. Plasma pharmacokinetics and renal excretion of paeoniflorin (5) in human subjects receiving an intravenous infusion of XueBiJing injection.

\begin{tabular}{|c|c|c|c|c|c|c|}
\hline \multirow{2}{*}{ PK parameter } & \multicolumn{2}{|c|}{$\begin{array}{l}\text { Dosage regimen for group A } \\
\text { (Paeoniflorin, } 114 \text { mg/subject) }\end{array}$} & \multicolumn{2}{|c|}{$\begin{array}{l}\text { Dosage regimen for group B } \\
\text { (Paeoniflorin, } 114 \text { mg/subject) }\end{array}$} & \multicolumn{2}{|c|}{$\begin{array}{l}\text { Dosage regimen for group C } \\
\text { (Paeoniflorin, } 57 \mathrm{mg} / \text { subject) }\end{array}$} \\
\hline & $\begin{array}{l}\text { Male } \\
(n=6)\end{array}$ & $\begin{array}{l}\text { Female } \\
(n=6)\end{array}$ & $\begin{array}{l}\text { Male } \\
(n=6)\end{array}$ & $\begin{array}{l}\text { Female } \\
(n=6)\end{array}$ & $\begin{array}{l}\text { Male } \\
(n=6)\end{array}$ & $\begin{array}{l}\text { Female } \\
(n=6)\end{array}$ \\
\hline \multicolumn{7}{|l|}{ Plasma data } \\
\hline$C_{\max }(\mu \mathrm{mol} / \mathrm{L})$ & $\begin{array}{l}18.2 \pm 5.7 \\
\text { (at } 75 \mathrm{~min})\end{array}$ & $\begin{array}{c}18.5 \pm 5.3 \\
\text { (at } 75 \mathrm{~min})\end{array}$ & $\begin{array}{c}11.1 \pm 3.0 \\
\text { (at } 150 \mathrm{~min})\end{array}$ & $\begin{array}{r}14.1 \pm 5.1^{\mathrm{b}} \\
(\text { at } 150 \mathrm{~min})\end{array}$ & $\begin{array}{c}9.27 \pm 1.80 \\
\text { (at } 75 \mathrm{~min})\end{array}$ & $\begin{array}{c}8.42 \pm 1.62 \\
\text { (at } 75 \mathrm{~min} \text { ) }\end{array}$ \\
\hline$t_{1 / 2}(\mathrm{~h})$ & $1.11 \pm 0.10$ & $1.08 \pm 0.08$ & $1.11 \pm 0.11$ & $1.16 \pm 0.13$ & $1.13 \pm 0.16$ & $1.09 \pm 0.11$ \\
\hline MRT (h) & $1.80 \pm 0.35$ & $1.46 \pm 0.13$ & $1.62 \pm 0.20$ & $1.66 \pm 0.22$ & $1.48 \pm 0.33$ & $1.54 \pm 0.14$ \\
\hline $\mathrm{CL}_{\text {tot, }, \mathrm{p}}\left(\mathrm{L}^{-1} \mathrm{~h}^{-1} \cdot \mathrm{kg}^{-1}\right)$ & $0.11 \pm 0.03$ & $0.13 \pm 0.03$ & $0.12 \pm 0.02$ & $0.12 \pm 0.02$ & $0.10 \pm 0.02$ & $0.13 \pm 0.02^{b}$ \\
\hline$V_{\mathrm{SS}}(\mathrm{L} / \mathrm{kg})$ & $0.19 \pm 0.05$ & $0.18 \pm 0.05$ & $0.20 \pm 0.04$ & $0.20 \pm 0.06$ & $0.15 \pm 0.03$ & $0.20 \pm 0.03^{b}$ \\
\hline$C_{\mathrm{SS}}(\mu \mathrm{mol} / \mathrm{L})$ & $28.2 \pm 7.6$ & $30.0 \pm 9.1$ & $12.8 \pm 2.4$ & $16.3 \pm 4.1$ & $14.7 \pm 1.8$ & $13.6 \pm 2.5$ \\
\hline$f_{\mathrm{e}-\mathrm{u}}(\%)$ & $62.5 \pm 6.5$ & $67.7 \pm 9.4$ & $58.7 \pm 10.9$ & $60.1 \pm 19.0$ & $59.1 \pm 9.6$ & $62.1 \pm 10.5$ \\
\hline $\mathrm{CL}_{\mathrm{R}} /\left(\mathrm{GFR} \times f_{\mathrm{u}}\right)$ & $0.73 \pm 0.07$ & $0.90 \pm 0.15$ & $0.75 \pm 0.10$ & $0.73 \pm 0.19$ & $0.65 \pm 0.13$ & $0.89 \pm 0.11$ \\
\hline
\end{tabular}

$C_{\max }$, finally measured plasma concentration just before stopping an infusion; $\mathrm{AUC}_{0-\infty}$, area under the plasma concentration-time curve from zero to infinity; $t_{1 / 2}$, elimination half-life; MRT, mean residence time; $\mathrm{CL}_{\text {tot,p }}$, total plasma clearance; $V_{\mathrm{sS}}$, apparent volume of distribution at steady state; $C_{\mathrm{sS}}$, calculated plasma concentration at steady state; $A_{S S}$, calculated plasma amount at steady state; $C u m . A_{e-u}$, cumulative amount excreted into urine; $\mathrm{CL}_{R}$, renal clearance; $f_{\mathrm{e}-u}$, fraction of dose excreted into urine; GFR, glomerular filtration rate. ${ }^{\mathrm{b}} \mathrm{P}<0.05$.

Dosage regimen for group A: a 75-min infusion of 100-mL XueBiJing injection (diluted in $100 \mathrm{~mL}$ of $0.9 \% \mathrm{NaCl}$ injection).

Dosage regimen for group B: a 150-min infusion of 100-mL XueBiJing injection (diluted in $200 \mathrm{~mL}$ of $0.9 \% \mathrm{NaCl}$ injection).

Dosage regimen for group C: a 75 -min infusion of 50-mL XueBiJing injection (diluted in $100 \mathrm{~mL}$ of $0.9 \% \mathrm{NaCl}$ injection).

Table 3. Plasma pharmacokinetics of paeoniflorin (5) in rats receiving a 30-min intravenous infusion of XueBiJing injection.

\begin{tabular}{|c|c|c|c|}
\hline PK parameter & $\begin{array}{c}5 \mathrm{~mL} / \mathrm{kg} \\
\text { (Paeoniflorin, } \\
5.7 \mathrm{mg} / \mathrm{kg} \text { ) }\end{array}$ & $\begin{array}{c}10 \mathrm{~mL} / \mathrm{kg} \\
\text { (Paeoniflorin, } \\
11.4 \mathrm{mg} / \mathrm{kg} \text { ) }\end{array}$ & $\begin{array}{c}20 \mathrm{~mL} / \mathrm{kg} \\
\text { (Paeoniflorin } \\
22.8 \mathrm{mg} / \mathrm{kg} \text { ) }\end{array}$ \\
\hline$C_{30 \min }(\mu \mathrm{mol} / \mathrm{L})$ & $24.6 \pm 5.0$ & $48.6 \pm 3.6$ & $95.3 \pm 16.8$ \\
\hline $\mathrm{AUC}_{0-\infty}(\mu \mathrm{mol} / \mathrm{L} \cdot \mathrm{h})$ & $13.8 \pm 3.2$ & $28.4 \pm 3.7$ & $56.0 \pm 9.8$ \\
\hline$t_{1 / 2}(\mathrm{~h})$ & $0.37 \pm 0.15$ & $0.43 \pm 0.09$ & $0.47 \pm 0.21$ \\
\hline MRT (h) & $0.55 \pm 0.05$ & $0.56 \pm 0.03$ & $0.58 \pm 0.04$ \\
\hline $\mathrm{CL}_{\text {tot, } \mathrm{p}}\left(\mathrm{L} \cdot \mathrm{h}^{-1} \cdot \mathrm{kg}^{-1}\right)$ & $0.90 \pm 0.22$ & $0.85 \pm 0.11$ & $0.87 \pm 0.16$ \\
\hline$V_{\mathrm{SS}}(\mathrm{L} / \mathrm{kg})$ & $0.26 \pm 0.02$ & $0.26 \pm 0.02$ & $0.31 \pm 0.06$ \\
\hline
\end{tabular}

$C_{30 \text { min }}$, concentration at 30 min after dosing; $\mathrm{AUC}_{0-\infty}$, area under the plasma concentration-time curve from zero to infinity; $t_{1 / 2}$, elimination half-life; MRT, mean residence time; $\mathrm{CL}_{\text {tot,p }}$, total plasma clearance; $V_{\mathrm{SS}}$, apparent volume of distribution at steady state.

injections normally contain multiple pharmacologically active compounds together with complex matrix components. Figure $4 \mathrm{~B}$ shows comparative rat plasma concentration-time curves of 5 (after intravenous dosing of XueBijing injection) and paeoniflorin (after intravenous dosing of the pure compound). The PK parameters of 5 were not significantly different from those of purified paeoniflorin $(P=0.1-1.0$; Table 6$)$, suggesting that other compounds present in the injection had a limited influence on the pharmacokinetics of 5 . After an intravenous bolus
Table 4. Renal and hepatic excretion of paeoniflorin (5) in rats receiving a 30-min intravenous infusion of XueBiJing injection at $10 \mathrm{~mL} / \mathrm{kg}$.

\begin{tabular}{ll}
\hline PK parameter & $10 \mathrm{~mL} / \mathrm{kg}$ (Paeoniflorin, $11.4 \mathrm{mg} / \mathrm{kg})$ \\
\hline Urine data & \\
Cum. $A_{\mathrm{e} \cdot \mathrm{U}}(\mu \mathrm{mol})$ & $3.07 \pm 0.51$ \\
$\mathrm{CL}_{\mathrm{R}}\left(\mathrm{L} \cdot \mathrm{h}^{-1} \cdot \mathrm{kg}^{-1}\right)$ & $0.42 \pm 0.07$ \\
$f_{\mathrm{e} \cdot \mathrm{U}}(\%)$ & $50.2 \pm 8.9$ \\
$\mathrm{CL}_{\mathrm{R}} /\left(\mathrm{GFR} \times f_{\mathrm{u}}\right)$ & $1.43 \pm 0.25$ \\
Bile data & \\
Cum. $A_{\mathrm{e} \cdot \mathrm{B}}(\mu \mathrm{mol})$ & $0.18 \pm 0.05$ \\
$\mathrm{CL} L_{\mathrm{B}}\left(\mathrm{L} \cdot \mathrm{h}^{-1} \cdot \mathrm{kg}^{-1}\right)$ & $0.03 \pm 0.01$ \\
$f_{\mathrm{e}-\mathrm{B}}(\%)$ & $3.34 \pm 0.94$ \\
\hline
\end{tabular}

Cum. $A_{\mathrm{e}-u}$, cumulative amount excreted into urine; $\mathrm{CL}_{\mathrm{R}}$, renal clearance; $f_{\mathrm{e}-\mathrm{u}}$, fraction of dose excreted into urine; GFR, glomerular filtration rate; Cum. $A_{\mathrm{e}-\mathrm{B}}$, cumulative amount excreted into bile; $\mathrm{CL}_{\mathrm{B}}$, biliary clearance; $f_{\mathrm{e}-\mathrm{B}}$, fraction of dose excreted into bile.

dose of the herbal injection, 5 was rapidly distributed into the rat tissues, including the lungs, heart, liver, kidneys, and brain. This was indicated by the highest tissue concentrations of 5 , which occurred at the first measured time point (5 min after dosing). Consistent with the small apparent distribution volume of 5, the exposure levels in the tissues were lower than the corresponding plasma levels, except for the kidney level, which was 4.3-times as much as the plasma level (Table 7). 
Table 5. Results from dose proportionality assessment for plasma paeoniflorin (5) in rats receiving a 30-min intravenous infusion of XueBiJing injection at 5,10 , and $20 \mathrm{~mL} / \mathrm{kg}$.

\begin{tabular}{lcccl}
\hline PK parameter & $r$ & $P$ & Slope $(90 \% \mathrm{Cl})$ & Conclusion \\
\hline Plasma $C_{\max }$ & 0.96 & 0.000000000002 & $0.98(0.87-1.09)$ & Linear \\
Plasma AUC $_{0-\infty}$ & 0.96 & 0.00000000001 & $1.02(0.90-1.14)$ & Linear \\
\hline
\end{tabular}

Critical intervals were $0.84-1.16$ for the plasma data with XueBiJing injection. The term $r$ denotes the correlation coefficient. Correlations were statistically significant with a $P<0.05$. The term "linear" was concluded statistically if the $90 \%$ confidence interval $(90 \% \mathrm{Cl}$ ) for slope was contained completely within the critical interval; "inconclusive" was concluded statistically if the $90 \% \mathrm{Cl}$ lay partly within the critical interval; "nonlinear" was concluded statistically if the $90 \% \mathrm{Cl}$ was entirely outside the critical interval.

Due to its poor membrane permeability, 5 exhibited low brain penetration. The half-lives of 5 in the tissues $(0.18-0.35 \mathrm{~h})$ were short and comparable with those in plasma. Hepatobiliary excretion of $\mathbf{5}$ after dosing XueBijing injection was poor in rats (Table 4). According to the sum of $f_{\mathrm{e}-\mathrm{U}}$ and $f_{\mathrm{e}-\mathrm{B} \text {, the overall }}$ recovery of $\mathbf{5}$ from intravenously dosed XueBiJing injection in rats was estimated to be $53.5 \%$. In the current study, no significant metabolite of 5 was detected in human subjects and rats receiving the injection; whether additional elimination pathway(s) exist for 5 remains to be elucidated.

\section{Discussion}

Many herbal products are used as medicines in China, because they exert therapeutic actions in clinics ${ }^{[5-8,55-58]}$. Further analysis of the therapeutic actions of an herbal medicine provides the basis for both the rational therapeutic use of the medicine and the design of new and superior therapeutic agents. To this end, it is important to identify the active principles responsible for the therapeutic actions of herbal medicines and the mechanisms of action. PK research on herbal medicines can facilitate the investigation of the medicines' therapeutic actions in different ways. First, unlike synthetic drugs, herbal medicines normally contain multiple bioactive constituents with different content levels and physicochemical characteristics. In addition to their pharmacological properties and potency, the reachability of herbal constituents for and their concentrations at the sites of action also determine which of them are responsible for the therapeutic actions of the dosed
Table 6. Comparative plasma pharmacokinetics of paeoniflorin (5) in rats receiving an intravenous bolus dose of XueBiJing injection at 10 $\mathrm{mL} / \mathrm{kg}$ (each milliliter of injection containing $1.14 \mathrm{mg}$ of (5) or purified paeoniflorin at $11.4 \mathrm{mg} / \mathrm{kg}$ (paeoniflorin being dissolved in vehicle of XueBiJing injection).

\begin{tabular}{lcc}
\hline PK parameter & XueBiJing injection & Purified paeoniflorin \\
\hline$C_{5 \min }(\mu \mathrm{mol} / \mathrm{L})$ & $72.8 \pm 2.6$ & $68.5 \pm 9.0$ \\
$\mathrm{AUC}_{0-\infty}(\mu \mathrm{mol} / \mathrm{L} \cdot \mathrm{h})$ & $26.3 \pm 4.2$ & $26.3 \pm 4.1$ \\
$t_{1 / 2}(\mathrm{~h})$ & $0.26 \pm 0.03$ & $0.26 \pm 0.01$ \\
$\mathrm{MRT}(\mathrm{h})$ & $0.36 \pm 0.05$ & $0.37 \pm 0.04$ \\
$\mathrm{CL}_{\text {tot, },}\left(\mathrm{L} \cdot \mathrm{h}^{-1} \cdot \mathrm{kg}^{-1}\right)$ & $0.92 \pm 0.16$ & $0.80 \pm 0.11$ \\
$V_{\mathrm{SS}}(\mathrm{L} / \mathrm{kg})$ & $0.24 \pm 0.02$ & $0.21 \pm 0.03$ \\
\hline
\end{tabular}

$\mathrm{C}_{5 \mathrm{~min}}$, concentration at $5 \mathrm{~min}$ after dosing; $\mathrm{AUC}_{0-\infty}$, area under the plasma concentration-time curve from zero to infinity; $t_{1 / 2}$, elimination half-life; $\mathrm{MRT}$, mean residence time; $\mathrm{CL}_{\text {tot,p }}$, total plasma clearance; $V_{\mathrm{sS}}$, apparent volume of distribution at steady state. Paeoniflorin was dissolved in the vehicle that was used to prepare XueBiJing injection.

medicine. Systemic exposure to herbal constituents after dosing a medicine is a prerequisite for their access to the action sites; the changes in the levels of systemic exposure normally reflect the changes in the concentrations at the sites of action. In addition, biotransformation, which often occurs mainly in the liver, can alter the forms of the exposure to the compounds and their reachability for the action sites. Accordingly, the assessment of systemic exposure to the herbal constituents, by measuring both their unchanged compounds and metabolites, is a key step in identifying the active principles responsible for the therapeutic action of the administered medicine. Second, for an herbal medicine, the mechanisms of action often involve the interactions of its circulating constituents and/or metabolites with receptors. Herbal compounds can distribute differentially into intracellular and/or extracellular (interstitial and plasma) compartments; such differential distribution is determined by their PK properties, including the membrane permeability and binding to macromolecular components in the blood. Additionally, the location of receptors can be either extracellular or intracellular. Although a receptor can be a drug target, both the interaction of an herbal compound with this receptor (as observed in vitro) and the receptor itself (when related to the compound) may not be important for the therapeutic action, if the compound can not reach the receptor in vivo. Accordingly, the assessment of the distribution fea-

Table 7. Tissue penetration of paeoniflorin (5) after an intravenous bolus dose of XueBiJing injection at $10 \mathrm{~mL} / \mathrm{kg}$ in rats.

\begin{tabular}{llllll}
\hline PK parameter & Lung & Heart & Brain & Kidney & Liver \\
\hline$C_{\max }(\mu \mathrm{mol} / \mathrm{L})$ & $28.1 \pm 2.5$ & $16.4 \pm 1.3$ & $0.89 \pm 0.05$ & $378 \pm 114$ & $25.4 \pm 7.4$ \\
$\mathrm{AUC}_{0-\infty}(\mu \mathrm{mol} / \mathrm{L} \cdot \mathrm{h})$ & $12.0 \pm 1.5$ & $6.55 \pm 0.85$ & $0.43 \pm 0.08$ & $121 \pm 24$ & $80.3 \pm 3.6$ \\
$t_{1 / 2}(\mathrm{~h})$ & $0.30 \pm 0.02$ & $0.27 \pm 0.01$ & $0.33 \pm 0.02$ & $0.20 \pm 0.00$ & $0.26 \pm 0.04$ \\
\hline
\end{tabular}

$C_{\max }$, concentration at 5 min after dosing for heart, lung, brain, kidney, and plasma and concentration at 15 min after dosing for liver; $\mathrm{AUC}_{0-\infty}$, area under the tissue or plasma concentration-time curve from zero to infinity; $t_{1 / 2}$, half-life. 
tures of circulating herbal compounds and their PK properties governing distribution is helpful for judging the importance of an interaction with a receptor for the action mechanism of the medicine.

Four classes of bioactive herbal constituents may be important for the therapeutic action of XueBiJing injection-flavonoids originating from Honghua, monoterpene glycosides from Chishao, phthalides from Chuanxiong and Danggui, and catechols from Danshen. PK research on XueBijing injection was designed to identify which of the bioactive herbal compounds (unchanged and metabolized) exhibit significant systemic exposure after dosing the injection and to characterize the major circulating compounds with respect to PK properties and profiles. As a part of the PK research on XueBijing injection, the current study focused on the Chishao monoterpene glycosides and provides some important information regarding the herbal injection for pharmacologists and clinical researchers.

First, among the monoterpene glycosides originating from Chishao, paeoniflorin (5) is the only one that considerably circulates in human subjects receiving XueBiJing injection. Further investigation of this compound, as well as the major circulating XueBiJing compounds still to be identified from the other component herbs, will facilitate the identification of the chemical basis for the antiseptic actions of XueBiJing injection and a better understanding of the mechanisms of action.

Second, XueBiJing injection is derived from a commonly used oral herbal formula called Xuefu-Zhuyu-Tang, which is a decoction of an 11-herb combination (consisting of Honghua, Chishao, Chuanxiong, Danggui, and seven other herbs). It has been reported that orally administered paeoniflorin has poor bioavailability and is bio-transformed into multiple metabolites (including paeonimetabolin I, paeonimetabolin II, and paeoniflorgenin) by the intestinal microflora ${ }^{[37-45]}$. Unlike oral administration of Xuefu-Zhuyu-Tang, intravenous administration of XueBiJing injection results in unchanged paeoniflorin (5), rather than its metabolites, being the circulating form. Intravenously dosed 5 from XueBiJing injection can not be transformed into the metabolites by the intestinal microflora, because the compound is quite slowly excreted into rat bile and because it is not the substrate of the intestinal efflux ABC transporters. Accordingly, pharmacologists should investigate unchanged 5-based mechanisms of action to understand the antiseptic properties of XueBijing injection.

Third, the small distribution volume of paeoniflorin (5) and its poor membrane permeability indicate that the compound interacts mainly with extracellular receptors to elicit its pharmacological effects. Unlike the other tested tissues, rat kidneys exhibited much higher apparent homogenate concentrations of 5 than the respective plasma concentrations. The filtered water was 99\% reabsorbed in the nephron tubular lumen, whereas 5 in the filtrate was poorly reabsorbed; this resulted in 5 being substantially concentrated in the tubular lumen. Accordingly, the distribution of $\mathbf{5}$ in the kidneys is expected to also be in the extracellular fluid compartments.

Fourth, several cell-based studies suggested that paeoni- florin isolate exhibits neuroprotective effects ${ }^{[30-33]}$. However, paeoniflorin (5) was found to have poor brain penetration in the current study. The translation of the neuropharmacological findings from the laboratory to the clinic is most likely impeded by the compound's limited brain delivery.

Fifth, glomerular-filtration-based renal excretion is the major elimination pathway of paeoniflorin (5) in humans and rats. Renal GFR and plasma levels of proteins (such as albumin and a1-acidglycoprotein) are two major influencing factors for glomerular-filtration-based renal excretion of a drug. The clinical phenotype of sepsis-induced acute kidney injury is characterized by profound decreases in GFR and creatinine clearance and development of uremia ${ }^{[59]}$. The sepsis-induced decrease in GFR is expected to lead to the decreased renal excretion of 5, and in turn, an increased level of systemic exposure to the compound. The release of hepatic plasma proteins changes when sepsis develops, and this includes decreased albumin level ${ }^{[60]}$. However, this may have limited influence on systemic exposure to 5 after dosing XueBiJing injection due to the compound's low binding to plasma protein.

Sixth, the level of rat systemic exposure to paeoniflorin (5) increases proportionally with increasing dose of XueBiJing injection, and this is expected to be similar in humans. This means that it is easy to predict the change in plasma concentration of paeoniflorin (5) in order to achieve the therapeutic objective when adjusting the dosage regimen of the injection.

Seventh, using the current dosage regimens with infusion times of 75 and 150 min for XueBiJing injection, the plateau concentrations $\left(C_{\mathrm{SS}}\right)$ of paeoniflorin (5) are never established during infusion. The $t_{1 / 2}$ of 5 is $1.2-1.3 \mathrm{~h}$ in human subjects, suggesting that over four hours of constant-rate infusion of the injection would be required before the compound's $C_{\mathrm{SS}}$ is reached. In clinics, XueBijing injection is given to patients with sepsis in the initial management that takes place within six hours of presentation. A delay exists between the start of an infusion and the maximum concentration of 5. A rational $C_{\mathrm{SS}}$ of $\mathbf{5}$ can be set according to the effective concentration of the compound's antiseptic property. Using the measured content level and estimated $\mathrm{CL}_{\text {tot, }}$ of 5 , an optimal infusion rate of XueBiJing injection can be calculated, and it will facilitate the translation of the pharmacological property of the compound to the overall antiseptic effect of the injection. In addition, to reach the $C_{\mathrm{SS}}$ more rapidly, a loading dose can be designed by giving an intravenous bolus dose of the injection at the start of infusion. The size of bolus dose is the compound's $A_{\mathrm{sS}}$, which can be calculated using the preceding Equation (3). It is worth mentioning that the final optimization of the dosage regimen of XueBijing injection for enhancement of its antiseptic efficacy should be based on the integration of the PK, antiseptic, and toxicological data of the injection's multiple active compounds. Accordingly, the PK research on XueBijing injection also includes characterization of pharmacokinetics and disposition of bioactive compounds originating from the other component herbs; the results are to be reported elsewhere.

In summary, paeoniflorin (5) was the predominant Chishao monoterpene glycoside present in XueBiJing injection, 
whereas the remaining 17 Chishao monoterpene glycosides were present at quite low content levels. Because of its notably high dose level, $\mathbf{5}$ was the only Chishao monoterpene glycoside with significant levels of systemic exposure in healthy human subjects and rats receiving the injection; the remaining monoterpene glycosides exhibited quite low exposure levels. Despite its low clearance, 5 resided in the extracellular fluid compartments in both species, mainly due to its poor membrane permeability; the very small distribution volume resulted in the compound having short elimination half-lives. No significant metabolite of $\mathbf{5}$ was detected in either species after dosing. Glomerular-filtration-based renal excretion was the major elimination pathway of $\mathbf{5}$, which was bound to plasma protein in a limited manner. The level of systemic exposure to 5 in rats increased in a manner proportional to the dose of XueBijing injection and was influenced in a limited manner by other compounds contained in the injection; similar scenarios are expected to take place in humans. After dosing XueBijing injection in rats, 5 exhibited lower levels of lung, heart, and liver exposure than the plasma level; its brain penetration was poor. However, its level of kidney exposure was significantly higher than the plasma level. Pharmacologically active paeoniflorin is a promising ingredient of therapeutic importance for XueBijing injection due to its significant systemic exposure and appropriate PK profiles after dosing the injection. This compound merits special consideration for further investigation on XueBijing injection, including PK/PD correlation studies of paeoniflorin and PK studies of the injection in patients with sepsis.

\section{Acknowledgements}

This work was funded by grants from the National Science \& Technology Major Project of China 'Key New Drug Creation and Manufacturing Program' (2009ZX09304-002 and 2011ZX09201-201-23), the National Science Foundation of China for Distinguished Young Scholars (30925044), the National Basic Research Program of China (2012CB518403), the National Natural Science Foundation of China (81503345), and the China Postdoctoral Science Foundation funded project (2014M560169).

\section{Author contribution}

Chuan LI, Chen CHENG, Yan SUN, and Yu-hong HUANG designed the research; Chen CHENG, Jia-zhen LIN, Li LI, Jun-ling YANG, Wei-wei JIA, Fei-fei DU, Feng-qing WANG, Mei-juan LI, Yan-fen LI, Fang XU, Na-ting ZHANG, Olajide E. OLALEYE, Yan SUN, and Jian LI performed the research; Chang-hai SUN and Gui-ping ZHANG contributed new reagents; Chuan LI, Chen CHENG, and Jia-zhen LIN analyzed data; and Chuan LI and Chen CHENG wrote the paper.

\section{Supplementary information}

Supplementary information is available at the Acta Pharmacologica Sinica's website.

\section{References}

1 Angus DC, van der Poll T. Severe sepsis and septic shock. N Engl J Med 2013; 369: 840-51.

2 Dellinger RP, Levy MM, Rhodes A, Annane D, Gerlach H, Opal SM, et al. Surviving sepsis campaign: international guidelines for management of severe sepsis and septic shock: 2012. Crit Care Med 2013; 41: 580-637.

3 Paul M, Shani V, Muchtar E, Kariv G, Robenshtok E, Leibovici L. Systematic review and meta-analysis of the efficacy of appropriate empiric antibiotic therapy for sepsis. Antimicrob Agents Chemother 2010; 54: 4851-63.

4 Fink MP, Warren HS. Strategies to improve drug development for sepsis. Nat Rev Drug Discov 2014; 13: 741-58.

5 Hu J, Shang HC, Li J, Zhang JH, Zhang L, Zhang BL. XueBiJing injection for sepsis: a comprehensive review. Med J Chin PLA 2010; 35: 9-12.

6 Li N, Jiang LW, Yu L, Zhang WH. Systematic review of XueBiJing injection for the treatment of sepsis. Chin J Mod Drug Appl 2013; 7: 8-11.

7 Li Q, Ren J, Liu G. Meta-analysis of XueBiJing in treating sepsis. Drug Eval 2014; 10: 9-13.

8 Hou SY, Feng XH, Lin CL, Tan YF. Efficacy of XueBiJing for coagulopathy in patients with sepsis. Saudi Med J 2015; 36: 164-9.

9 Lu T, Yang JL, Gao XM, Chen P, Du FF, Sun Y, et al. Plasma and urinary tanshinol from Salvia miltiorrhiza (Danshen), can be used as pharmacokinetic markers for cardiotonic pills, a cardiovascular herbal medicine. Drug Metab Dispos 2008; 36: 1578-86.

10 Jia WW, Du FF, Liu XW, Jiang RR, Xu F, Yang JL, et al. Renal tubular secretion of tanshinol: molecular mechanisms, impact on its systemic exposure, and propensity for dose-related nephrotoxicity and for renal herb-drug interactions. Drug Metab Dispos 2015; 43: 669-78.

11 Liu HF, Yang JL, Du FF, Gao XM, Ma XT, Huang YH, et al. Absorption and disposition of ginsenosides after oral administration of Panax notoginseng extract to rats. Drug Metab Dispos 2009; 37: 2290-8.

12 Li L, Zhao YS, Du FF, Yang JL, Xu F, Niu W, et al. Intestinal absorption and presystemic elimination of various chemical constituents present in GBE50 extract, a standardized extract of Ginkgo biloba leaves. Curr Drug Metab 2012; 13: 494-509.

13 Hu ZY, Yang JL, Cheng C, Huang YH, Du FF, Wang FQ, et al. Combinatorial metabolism notably affects human systemic exposure to ginsenosides from orally administered extract of Panax notoginseng roots (Sanqi). Drug Metab Dispos 2013; 41: 1457-69.

14 Cheng C, Liu XW, Du FF, Li MJ, Xu F, Wang FQ, et al. Sensitive assay for measurement of volatile borneol, isoborneol, and the metabolite camphor in rat pharmacokinetic study of Borneolum (Bingpian) and Borneolum syntheticum (synthetic Bingpian). Acta Pharmacol Sin 2013; 34: 1337-48.

15 Chen F, Li L, Xu F, Sun Y, Du FF, Ma XT, et al. Systemic and cerebral exposure to and pharmacokinetics of flavonols and terpene lactones after dosing standardized Ginkgo biloba leaf extracts to rats via different administration routes. Br J Pharmacol 2013; 170: 440-57.

16 Li MJ, Wang FQ, Huang YH, Du FF, Zhong CC, Olaleye OE, et al. Systemic exposure to and disposition of catechols derived from Salvia miltiorrhiza roots (Danshen) after intravenous administration of DanHong injection in human subjects, rats, and dogs. Drug Metab Dispos 2015; 43: 679-90.

17 Yan R, Ko NL, Ma B, Tam YK, Lin G. Metabolic conversion from co-existing ingredient leading to significant systemic exposure of Z-butylidenephthalide, a minor ingredient in Chuanxiong Rhizoma in 
rat. Curr Drug Metab 2012; 13: 524-34.

18 Huang H, Ji LX, Song SY, Wang J, Wei N, Jiang M, et al. Identification of the major constituents in XueBiJing injection by HPLC-ESI-MS. Phytochem Anal 2011; 22: 330-8.

19 Jiang WL, Chen XG, Zhu HB, Gao YB, Tian JW, Fu FH. Paeoniflorin inhibits systemic inflammation and improves survival in experimental sepsis. Basic Clin Pharmacol Toxicol 2009; 105: 64-71.

20 Cao WJ, Zhang W, Liu JJ, Wang Y, Peng XM, Lu DX, et al. Paeoniflorin improves survival in LPS-challenged mice through the suppression of TNF- $\alpha$ and IL-1 $\beta$ release and augmentation of IL-10 production. Int Immunopharmacol 2011; 11: 172-8.

21 Zhou HQ, Bian DF, Jiao XL, Wei ZF, Zhang HF, Xia YF, et al. Paeoniflorin protects against lipopolysaccharide-induced acute lung injury in mice by alleviating inflammatory cell infiltration and microvascular permeability. Inflamm Res 2011; 60: 981-90.

22 Li JZ, Wu JH, Yu SY, Shao QR, Dong XM. Inhibitory effects of paeoniflorin on lysophosphatidylcholine-induced inflammatory factor production in human umbilical vein endothelial cells. Int J Mol Med 2013; 31: 493-7.

23 Zhang MH, Feng L, Zhu MM, Gu JF, Wu C, Jia XB. Antioxidative and anti-inflammatory activities of paeoniflorin and oxypaeoniflorin on AGEs-induced mesangial cell damage. Planta Med 2013; 79: 131923.

24 Jiang M, Zhou M, Han YQ, Xing L, Zhao HZ, Dong LY. Identification of NF-KB inhibitors in Xuebijing injection for sepsis treatment based on bioactivity-integrated UPLC-Q/TOF. J Ethnopharmacol 2013; 147: 426-33.

25 Zhu X, Fang ZH. New monoterpene glycosides from the root cortex of Paeonia suffruticosa and their potential anti-inflammatory activity. Nat Prod Res 2014; 28: 301-5.

26 Ye JF, Duan HL, Yang XM, Yan WM, Zheng XX. Anti-thrombosis effect of paeoniflorin: evaluated in a photochemical reaction thrombosis model in vivo. Planta Med 2001; 67: 766-7.

27 Zhu M, Tang YP, Duan JA, Guo JM, Guo S, Su SL, et al. Roles of paeoniflorin and senkyunolide I in SiWu decoction on antiplatelet and anticoagulation activities. J Sep Sci 2010; 33: 3335-40.

28 Koo YK, Kim JM, Koo JY, Kang SS, Bae K, Kim YS. Platelet antiaggregatory and blood anti-coagulant effects of compounds isolated from Paeonia lactiflora and Paeonia suffruticosa. Pharmazie 2010; 6: 624-8.

29 Suh KS, Choi EM, Lee YS, Kim YS. Protective effect of albiflorin against oxidative-stress-mediated toxicity in osteoblast-like MC3T3-E1 cells. Fitoterapia 2013; 89: 33-41.

30 Mao QQ, Zhong XM, Feng CR, Pan AJ, Li ZY, Huang Z. Protective effects of paeoniflorin against glutamate-induced neurotoxicity in PC12 cell via antioxidant mechanisms and $\mathrm{Ca}^{2+}$ antagonism. Cell Mol Neurobiol 2010; 30: 1059-66.

31 Cao BY, Yang YP, Luo WF, Mao CJ, Han R, Sun X, et al. Paeoniflorin, a potent natural compound, protects PC12 cells from $\mathrm{MPP}^{+}$and acidic damage via autophagic pathway. J Ethnopharmacol 2010; 131: 1229.

32 Wang D, Wong HK, Feng HY, Zhang ZJ. Paeoniflorin, a natural neuroprotective agent, modulates multiple anti-apoptotic and proapoptotic pathways in differentiated PC12 cells. Cell Mol Neurobiol 2013; 33: 521-9.

33 Wang D, Tan QR, Zhang ZJ. Neuroprotective effects of paeoniflorin, but not the isomer albiflorin, are associated with the suppression of intracellular calcium and calcium/calmodulin protein kinase II in PC12 cells. J Mol Neurosci 2013; 51: 581-90.

34 Sheng YX, Li L, Wang CS, Li YY, Guo DA. Solid-phase extraction-liquid chromatographic method for the determination and pharmacokinetic studies of albiflorin and paeoniflorin in rat serum after oral administration of Si-Wu decoction. J Chromatogr B 2004; 806: 127 32.

35 Xia SM, Shen R, Sun XY, Shen LL, Yang YM, Ke Y, et al. Development and validation of a sensitive liquid chromatography-tandem mass spectrometry method for the determination of paeoniflorin in rat brain and its application to pharmacokinetic study. J Chromatogr B 2007; 857: 32-9.

36 Tong L, Wan MX, Zhou DD, Cao J, Zhu YH, Bi KS. LC-MS/MS determination and pharmacokinetic study of albiflorin and paeoniflorin in rat plasma after oral administration of Radix Paeoniae Alba extract and Tang-Min-Ling-Wan. Biomed Chromatogr 2010; 24: 1324-31.

37 Takeda S, Isono T, Wakui Y, Matsuzaki Y, Sasaki H, Amagaya S, et al. Absorption and excretion of paeoniflorin in rats. J Pharm Pharmacol 1995; 47: 1036-40.

38 Li YF, Wang M, Wang XY, Yu HS, Kang LP, Ma BP, et al. Pharmacokinetic properties of albiflorin and paeoniflorin after oral administration of pure compound, Radix Paeoniae alba extract and Danggui-Shaoyao-San extract to rats. J Asian Nat Prod Res 2011; 13 : 117-27.

39 Takeda S, Isono T, Wakui Y, Mizuhara Y, Amagaya S, Maruno M, et al. In-vivo assessment of extrahepatic metabolism of paeoniflorin in rats: relevance to intestinal floral metabolism. J Pharm Pharmacol 1997; 49: 35-9.

40 Liu ZQ, Jiang ZH, Liu L, Hu M. Mechanisms responsible for poor oral bioavailability of paeoniflorin: role of intestinal disposition and interactions with sinomenine. Pharm Res 2006; 23: 2768-80.

41 Hattori M, Shu YZ, Shimizu M, Hayashi T, Morita N, Kobashi K, et al. Metabolism of paeoniflorin and related compounds by human intestinal bacteria. Chem Pharm Bull 1985; 33: 3838-46.

42 Shu YZ, Hattori M, Akao T, Kobashi K, Kagei K, Fukuyama K, et al. Metabolism of Paeoniflorin and related compounds by human intestinal bacteria. II. Structures of 7S- and 7R-paeonimetabolines I and II formed by Bacteroides fragilis and Lactobacillus brevis. Chem Pharm Bull 1987; 35: 3726-33.

$43 \mathrm{He}$ JX, Akao T, Tani T. Influence of co-administered antibiotics on the pharmacokinetic fate in rats of paeoniflorin and its active metabolite paeonimetabolin-I from Shaoyao-Gancao-tang. J Pharm Pharmacol 2003; 55: 313-21.

44 Hsiu SL, Lin YT, Wen KC, Hou YC, Chao PD. A deglucosylated metabolite of paeoniflorin of the root of Paeonia lactiflora and its pharmacokinetics in rats. Planta Med 2003; 69: 1113-8.

45 Liang J, Xu F, Zhang YZ, Huang S, Zang XY, Xin Zhao, et al. The profiling and identification of the absorbed constituents and metabolites of Paeoniae Radix Rubra decoction in rat plasma and urine by the HPLC-DAD-ESI-IT-TOF-MS ${ }^{n}$ technique: A novel strategy for the systematic screening and identification of absorbed constituents and metabolites from traditional Chinese medicines. J Pharm Biomed Anal 2013; 83: 108-21.

46 Reagan-Shaw S, Nihal M, Ahmad N. Dose translation from animal to human studies revisited. FASEB J 2008; 22: 659-61.

47 Guo B, Li C, Wang GJ, Chen LS. Rapid and direct measurement of free concentrations of highly protein-bound fluoxetine and its metabolite norfluoxetine in plasma. Rapid Commun Mass Spectrom 2006; 20: 39-47.

48 Dai JY, Yang JL, Li C. Transport and metabolism of flavonoids from Chinese herbal remedy Xiaochaihu-tang across human intestinal Caco-2 cell monolayers. Acta Pharmacol Sin 2008; 29: 1086-93.

49 Li YF, Sun Y, Du FF, Yuan KH, Li C. Pulse gradient, large-volume injection, high-throughput ultra-performance liquid chromatographic/ tandem mass spectrometry bioanalysis for measurement of plasma 
amrubicin and its metabolite amrubicinol. J Chromatogr A 2008; 1193: 109-16.

50 Smith BP, Vandenhende FR, DeSante KA, Farid NA, Welch PA, Callaghan JT, et al. Confidence interval criteria for assessment of dose proportionality. Pharm Res 2000; 17: 1278-83.

51 Li SL, Song JZ, Choi FF, Qiao CF, Zhou Y, Han QB, et al. Chemical profiling of Radix Paeoniae evaluated by ultra-performance liquid chromatography/photo-diode-array/quadrupole time-of-flight mass spectrometry. J Pharm Biomed Anal 2009; 49: 253-66.

52 Liu EH, Qi LW, Li B, Peng YB, Li P, Li CY, et al. High-speed separation and characterization of major constituents in Radix Paeoniae Rubra in fast high-performance liquid chromatography coupled with diodearray detection and time-of-flight mass spectrometry. Rapid Commun Mass Spectrom 2009; 23: 119-30.

53 Toutain PL, Bousquet-Mélou A. Plasma clearance. J Vet Pharmacol Ther 2004; 27: 427-39.

54 Davies B, Morris T. Physiological parameters in laboratory animals and humans. Pharm Res 1993; 10: 1093-5.

55 Mader LS. Chinese herbal medicine clears US FDA Phase II Trials. HerbalGram 2010; 88: 58-9.
56 Jia YL, Huang FY, Zhang SK, Leung SW. Is Danshen (Salvia miltiorrhiza) dripping pill more effective than isosorbide dinitrate in treating angina pectoris? A systematic review of randomized controlled trials. Int J Cardiol 2012; 157: 330-40.

57 Li XL, Zhang J, Huang J, Ma AQ, Yang JF, Li WM, et al. A multicenter, randomized, double-blind, parallel-group, placebo-controlled study of the effects of qili qiangxin capsules in patients with chronic heart failure. J Am Coll Cardiol 2013; 62: 1065-72.

58 Shang HC, Zhang JH, Yao C, Liu BY, Gao XM, Ren M, et al. Qi-Shen-YiQi dripping pills for the secondary prevention of myocardial infarction: a randomised clinical trial. Evid Based Complement Alternat Med 2013; 2013: 738391.

59 Gomez H, Ince C, Backer DD, Pickkers P, Payen D, Hotchkiss J, et al. A unified theory of sepsis-induced acute kidney injury: inflammation, microcirculatory dysfunction, bioenergetics and the tubular cell adaptation to injury. Shock 2014; 41: 3-11.

60 Sganga G, Siegel JH, Brown G, Coleman B, Wiles CE, Belzberg H, et al. Reprioritization of hepatic plasma protein release in trauma and sepsis. Arch Surg 1985: 187-99. 